\title{
On n'attrape pas les mouches avec du vinaigre
}

Tapettes et autres pièges à mouches

You catch more flies with honey than with vinegar. Fly traps and other devices

Nicolas Césard et Anie Montigny

\section{CpenEdition}

\section{Journals}

Édition électronique

URL : http://journals.openedition.org/ethnoecologie/5774

DOI : $10.4000 /$ ethnoecologie. 5774

ISSN : 2267-2419

Éditeur

Laboratoire Eco-anthropologie et Ethnobiologie

Référence électronique

Nicolas Césard et Anie Montigny, «On n'attrape pas les mouches avec du vinaigre », Revue

d'ethnoécologie [En ligne], 16 | 2019, mis en ligne le 31 décembre 2019, consulté le 06 janvier 2020.

URL : http://journals.openedition.org/ethnoecologie/5774 ; DOI : 10.4000/ethnoecologie.5774

Ce document a été généré automatiquement le 6 janvier 2020.

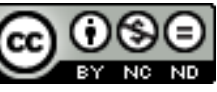

Revue d'ethnoécologie est mis à disposition selon les termes de la licence Creative Commons Attribution - Pas d'Utilisation Commerciale - Pas de Modification 4.0 International. 


\section{On n'attrape pas les mouches avec du vinaigre}

Tapettes et autres pièges à mouches

You catch more flies with honey than with vinegar. Fly traps and other devices

Nicolas Césard et Anie Montigny

«L'homme capable d'attraper une mouche avec
des baguettes peut tout accomplir » / Man who
catch fly with chopsticks accomplish anything-
Miyagi-sensei (Karaté Kid 1984)

\section{Introduction}

Chasse-mouches, tue-mouches, attrape-mouches, gobe-mouches, ces objets nous apparaissent d'abord par leur fonction, celle de l'utilité qu'on leur donne ou que l'on cherche à leur donner : éloigner, tuer ou piéger les insectes importuns ou indésirables. Une approche anthropocentrée, celle principalement de l'action cynégétique de l'homme sur l'animal, suffirait à les présenter. Une proposition complémentaire serait de mettre en avant une orientation plus biologique de l'objet, de sa conception à son utilisation, et de partir en grande partie de l'animal et de son milieu de vie pour comprendre à la fois la technique mise en œuvre et les attitudes à l'égard de l'insecte. Il s'agirait alors de concevoir et de rendre compte de l'objet moins comme une interface de contact, voire de mise à mort, qu'un temps et qu'un espace (physique) de rencontre entre espèces à l'issue plus incertaine. Dans cette perspective, la présentation qui suit s'intéresse à la manière dont les objets techniques peuvent être conçus à partir des animaux et dans nos relations avec eux, dans l'évolution de notre compréhension et de nos représentations d'une "catégorie " particulière d'insectes: les mouches domestiques. 


\section{Les mouches sont-elles chassées ou piégées?}

2 La réponse dépend en partie de la perspective adoptée. Au regard de la motivation de leurs propriétaires et des outils utilisés, les mouches sont le plus souvent chassées ou capturées pour être exterminées. Poursuivre les mouches pour les tuer apparaît comme une activité de chasse même s'il s'avère dans la pratique plus facile de les capturer. Mais le choix des méthodes par l'utilisateur relève autant de l'efficacité de l'engin que de l'opérateur : la chasse peut être considérée comme un exercice d'habileté, ou un jeu, alors que le piège fonctionne dans la durée et ne requiert pas les mêmes efforts. En anthropologie, l'activité de piégeage est définie par la présence facultative de l'homme et l'automatisme de la capture (Haudricourt \& Bert 2010). Serge Bahuchet distingue la chasse directe qui oppose l'homme à l'animal, de la chasse indirecte qui utilise des engins et des machines pour la capture du gibier (Bahuchet 1971). Or, le qualificatif de chasse, comme la distinction entre chasse et piégeage sur la base de la présenceabsence et de l'activité-passivité (Mauss 1947), reflètent la position du chasseurpiégeur, et non celle du chassé-piégé. Du point de vue de l'insecte, ou plutôt à son échelle, nous lui préférons ici l'appellation de piège car bien souvent, comme nous allons le voir, la mouche est piégée dans ses habitudes par le chasseur avant d'être tuée ou capturée (Figure 1).

Figure 1 : Carte postale humoristique ETB-UK-NC-2019-04

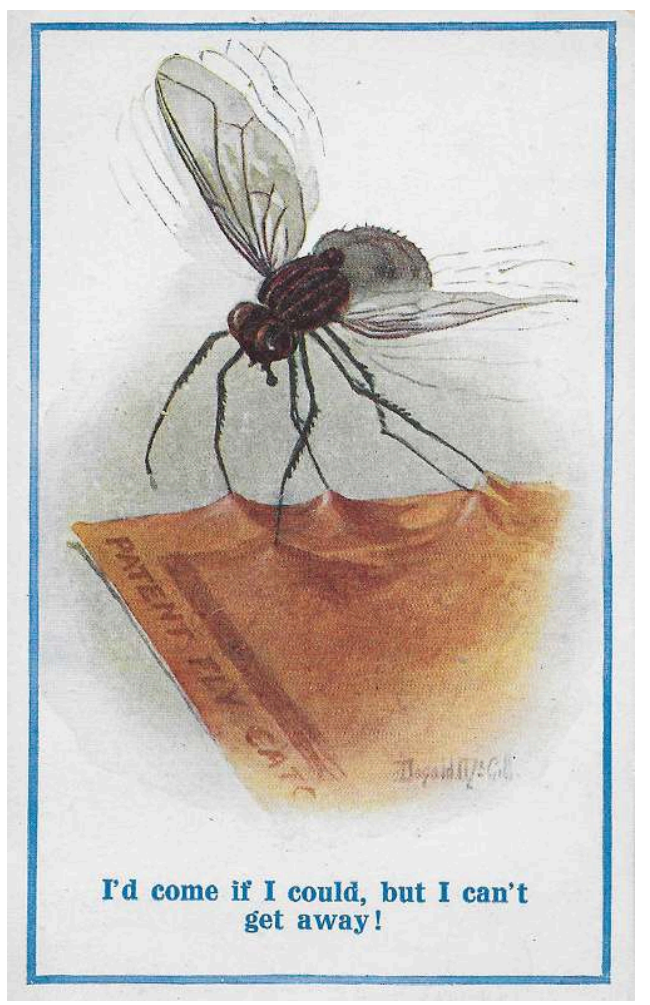

(c) N. Césard/MNHN

3 - Angleterre, datée du 27/04/1918

- « Je viendrais si je le pouvais mais je ne peux pas m'enfuir ! "

- Juillet 2019 ; collecteur Nicolas Césard 
- Dimensions (cm) : 9,5 x 14

- Nº d'inventaire : ETB-UK-NC-2019-04

\section{Des outils adaptés à la mouche}

4 Les objets présentés partent en grande partie du comportement de l'animal dans son milieu pour penser la technique. Leurs modes d'action sont conçus principalement à partir d'observations élémentaires et empiriques : celles du vol des mouches et de leur alimentation, en particulier leur attrait pour les aliments sucrés.

Des générations de chasseurs se sont interrogées sur la meilleure façon d'approcher les mouches ${ }^{2}$. Leurs observations ne s'appuient pas sur des informations scientifiques, comme c'est le cas pour le développement d'autres objets techniques, mais trouvent depuis peu des explications dans la science. La neurologie des insectes en particulier montre que les capacités des hommes et des mouches à percevoir le monde diffèrent. Les études récentes expliquent que si la mouche voit relativement mal, elle est capable d'analyser très rapidement les éléments qui l'entourent, notamment ceux en mouvement. Beaucoup plus rapide que celui de l'homme, son système visuel lui fait voir la menace humaine au ralenti : à la vue d'une tapette, la mouche s'immobilise en même temps qu'elle repositionne son corps, ses pattes et ses ailes pour s'envoler en moins d'un dixième de seconde loin de l'objet ${ }^{3}$ (VIDEO 1).

Vidéo 1. Fly Swat Science. Why are flies so hard to swat? https://www.youtube.com/watch? $v=$ otqcVG-nJGI

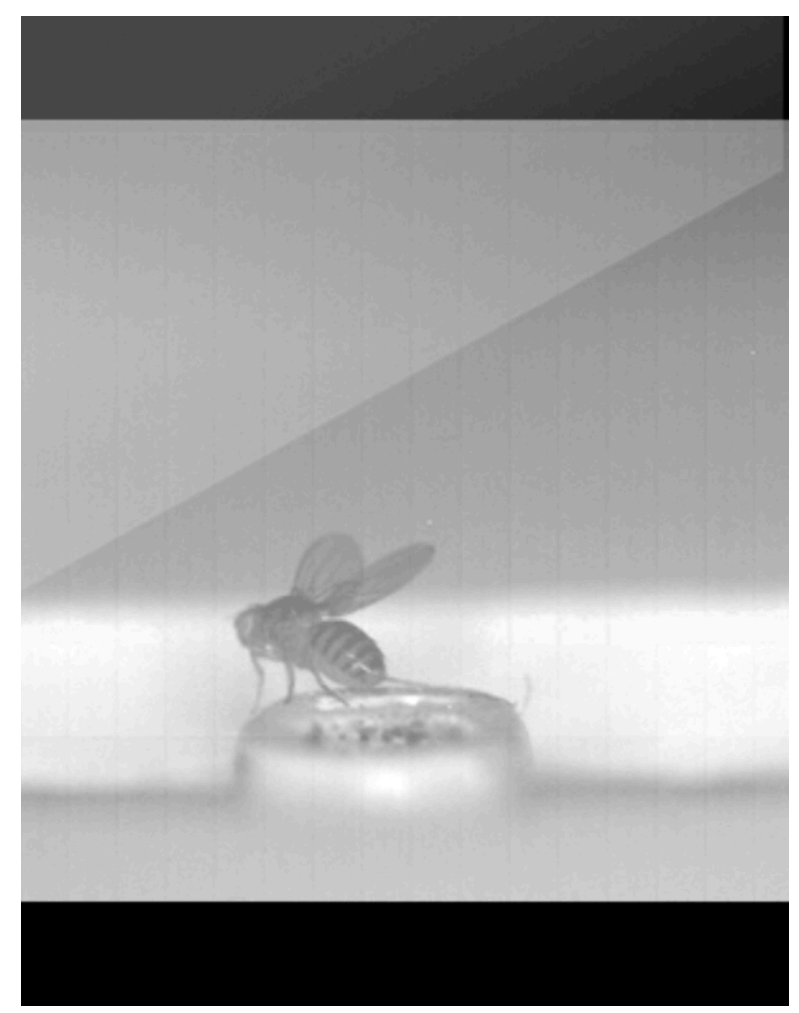

Source: Sciencentral / Gwyneth Card, Michael Dickinson, Current Biology, 28 aout, 2008

6 Si la mouche semble mieux dotée pour prédire les mouvements du chasseur que le chasseur ceux de sa proie, son interaction avec l'homme n'est pas toujours à son 
avantage. L'homme conçoit toutes sortes d'engins pour surprendre les mouches à l'arrêt ou en vol. Ces derniers nécessitent une certaine dextérité dans leur maniement et un minimum d'anticipation sur la mouche qui, avec le temps, permet au chasseur d'affiner sa connaissance des déplacements de l'insecte. Par ailleurs, le chasseur s'appuie sur un second comportement de la mouche pour la piéger : son appétit, une quête de nourriture suivie d'une phase d'activité en apparence plus statique qui la rend vulnérable aux attaques ou à sa capture. Ces deux comportements sont séparés ou associés mais apparaissent plus efficaces combinés dans une «convergence des fonctions » propre à l'objet (Simondon 1958).

7 Dans la continuité de Théodore Monod (1973) et de Serge Bahuchet et Pauline Rameau (2016), nous proposons à la suite une distinction des pièges par type de menace rencontré (par l'animal) plutôt que par fonction (humaine) de l'objet, une typologie fondée sur « la nature de l'action (principale) » (Monod 1973) portée à l'insecte plus que sur les procédés déployés. Comme pour d'autres objets techniques, chaque piège s'inscrit dans une évolution dont le sens tend vers une "individualisation ", la structure de celui-ci, son système technique, devenant le modèle d'autres artefacts, comme en témoigne l'évolution des pièges à travers les dépôts des brevets états-uniens.

\section{Pièges « éclairs » : tapettes et autres claque-mouches}

8 La première catégorie regroupe les pièges que nous appellerons pièges " éclairs ", c'està-dire ceux qui prennent de vitesse la mouche en lui opposant une surface plus ou moins large, qu'elle soit simple ou double. Ces derniers demandent d'aller au-devant de la mouche et donc de son déplacement. Ils intègrent des réponses perceptives et comportementales, voire l'élargissent à une certaine agentivité de l'insecte, mais ne réussissent pas toujours à le piéger.

9 Claquer des deux mains est un geste commun - mais assez peu efficace - pour attraper la mouche lors de l'envol et les premiers claque-mouches ou tapettes doubles brevetés aux États-Unis (connus alors comme fly catcher, insect catcher) s'en inspirent directement (Linding $1928 \mathrm{n}^{\circ} 1660011$; Voss $1928 \mathrm{n}^{\circ}$ 1664762). À ces derniers succèderont des tapettes dont les deux battoirs sont articulés par la pression d'un manche (Gustke $1940 \mathrm{n}^{\circ} 2191126$; Linding $1948 \mathrm{n}^{\circ} 2434364$; Sharpe $1951 \mathrm{n}^{\circ} 2545215$ ) (Figures 2 et 3 pour un modèle japonais) ou libérés à l'aide d'un piston (Figure 4). 
Figure 2 : Claque-mouches à deux battoirs - ETB-US-NC-2019-05

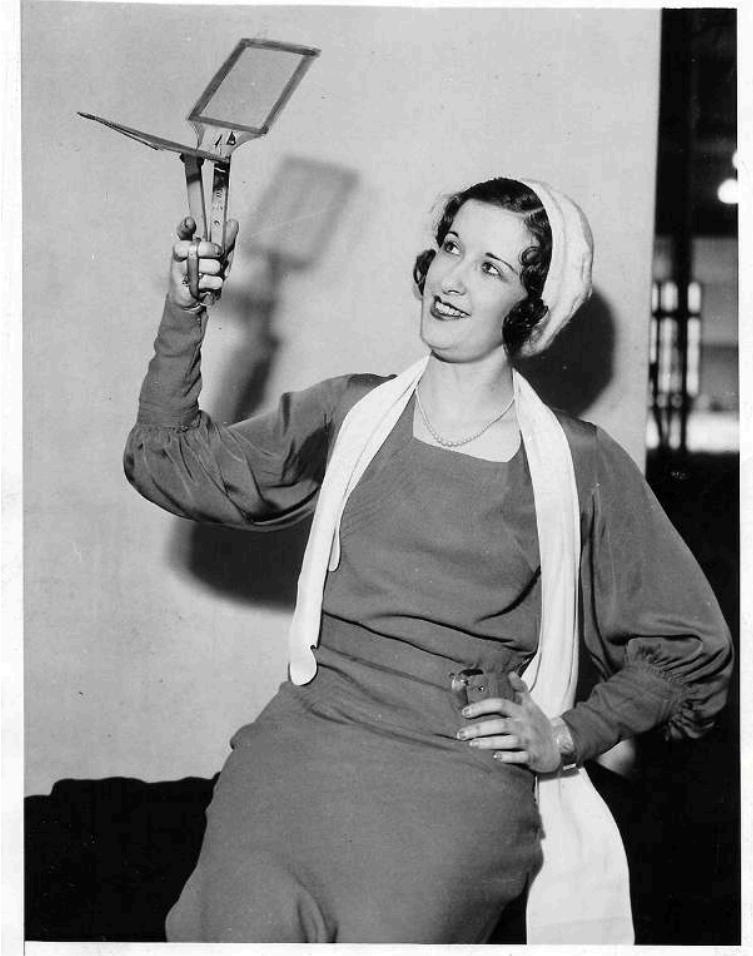

(c) N. Césard/MNHN

10 - États-Unis

- Photo de presse de la $4^{\text {ème }}$ exposition internationale de brevets montrant Helena Linding et le modèle de tapette à deux battoirs (fly swatter), New York, 1932

- Juin 2019 ; collecteur Nicolas Césard

- Dimensions (cm) : $20 \times 15$

- Nº d'inventaire : ETB-US-NC-2019-05 


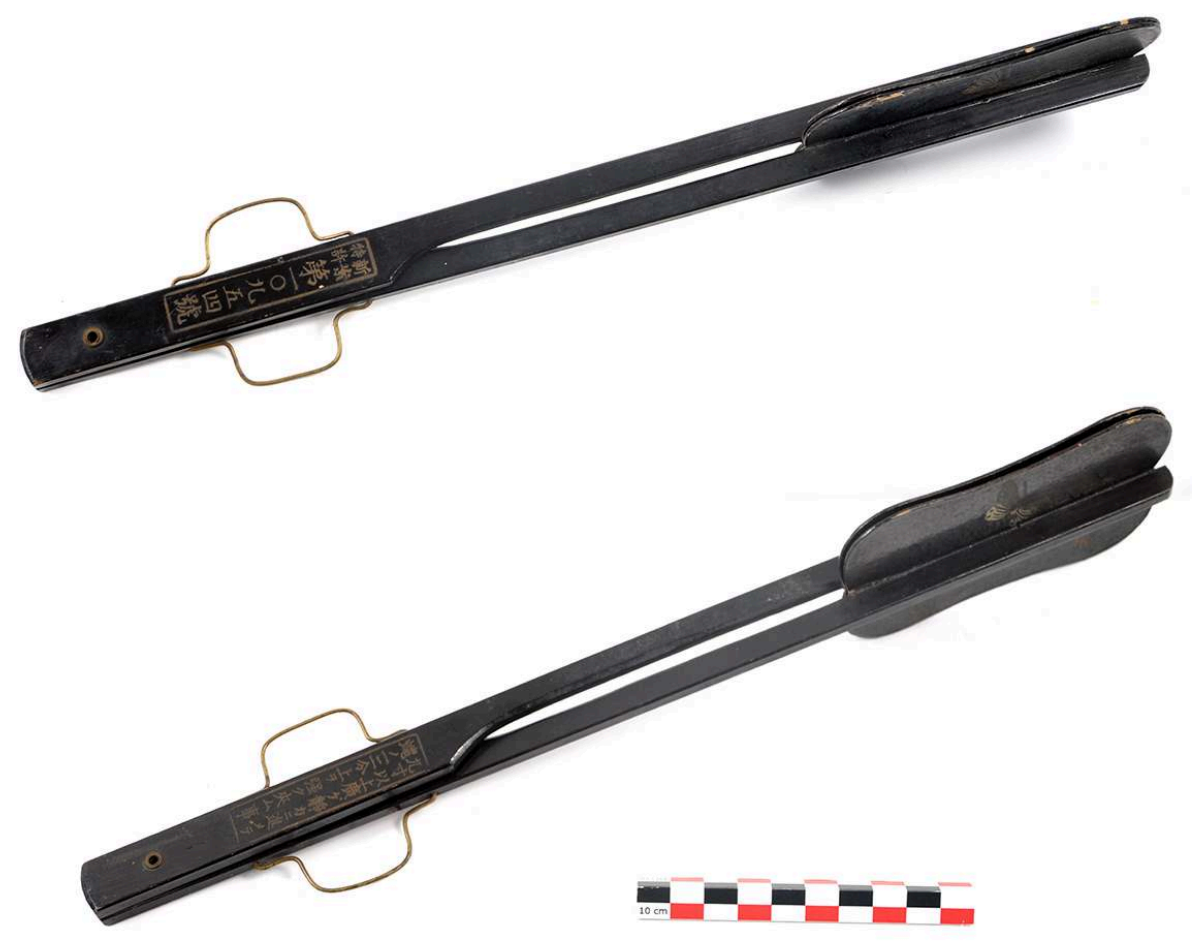

(c) M. Kourdourli/MNHN

11 - Japon; années 1950

- Objet en bois laqué et fer. Le manche permet d'actionner les deux battoirs comme une paire de ciseaux. Les battoirs sont décorés de papillons dorés sur leurs faces extérieures. Des instructions sont inscrites sur chaque côté du manche. Sur l'un, on peut lire : «On peut l'ouvrir sur plus de 9 pouces [20 centimètres] et l'avancer sans faire de bruit. On peut pincer fortement plus de $30 \%$ de mouches ». (Traduction Nathalie Allain).

- Octobre 2018 ; collecteur Nicolas Césard

- Dimensions $(\mathrm{cm}): 34,5 \times 7$

- $\mathrm{N}^{\circ}$ d'inventaire : ETB-JP-NC-2018-037 
Figure 4 : Claque-mouches à deux battoirs ETB-UK-NC-2019-02

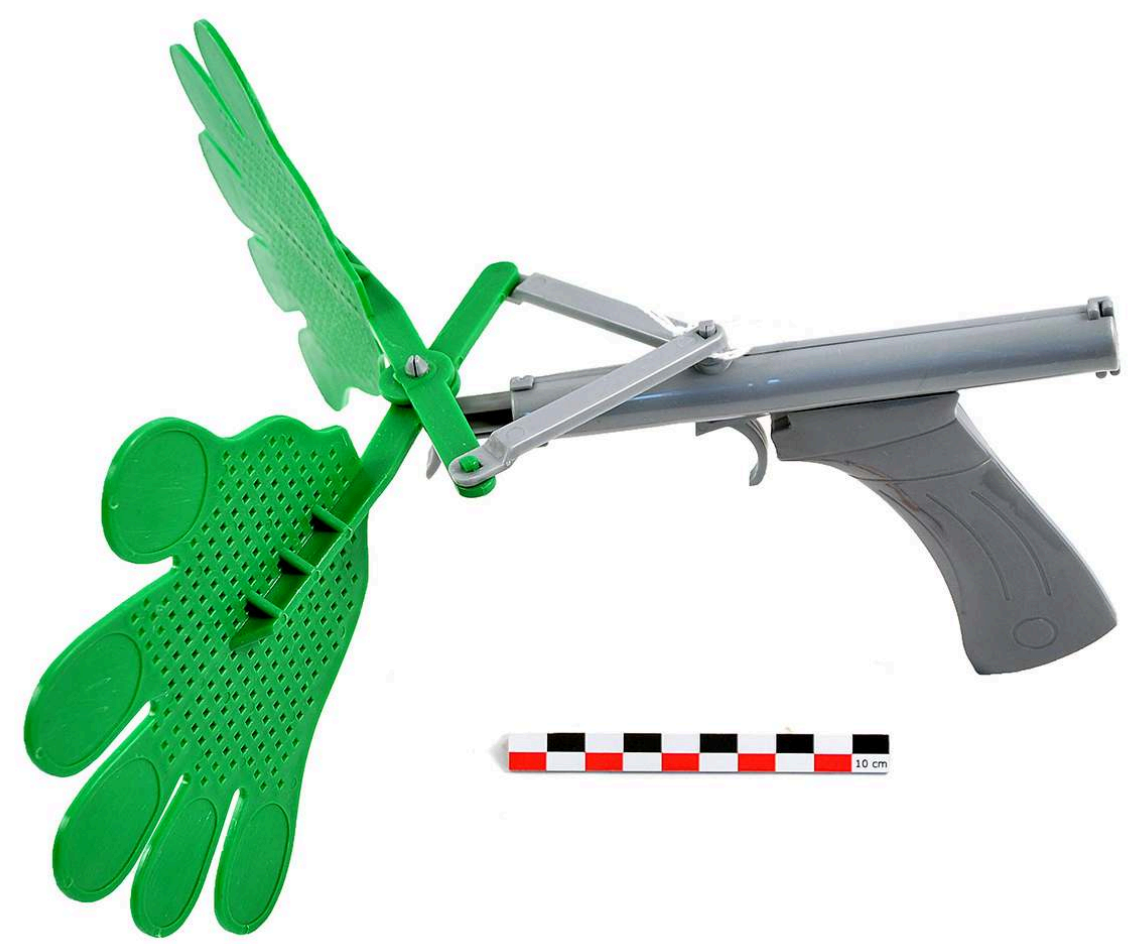

(c) M. Kourdourli/MNHN

12 - Royaume-Uni ; 2019

- Claque-mouches à deux battoirs (fly clapper pistol)

- Juillet 2019 ; collecteur Nicolas Césard

- Dimensions (cm) : 38 x 20 (fermé), 28 x 28 (ouvert)

- $\mathrm{N}^{\circ}$ d'inventaire : ETB-UK-NC-2019-02

La tapette à mouches à un seul battoir ou tapette simple est l'objet le plus connu et le plus populaire des pièges « éclairs ». Instrument de chasse pour les hommes, piège pour l'insecte, elle bloque la mouche au moment de son envol pour la rabattre et l'écraser entre son tamis et toute autre surface, tel un mur ou une table. Son efficacité dépend de sa conception comme de l'habilité directe de celui qui l'utilise. D'apparition plus tardive, les raquettes électriques existent en plusieurs modèles et répondent au même principe de capture: un tamis surprend la mouche dans son vol et à son contact l'électrifie. La première raquette électrique brevetée aux États-Unis (alors nommée fly swatter, Laine 1959 brevet $n^{\circ} 2881554$ ) demande moins d'efforts par rapport à une tapette classique mais promet aussi d'être plus propre: "une mouche ou tout autre insecte peut être tuée sans l'écraser sous l'impact contre un support ferme ou solide ».

14 À noter que la tapette, simple ou double, bénéficie souvent indirectement d'un appât qui maintient la mouche occupée ou à l'arrêt, comme un résidu alimentaire, ou d'un leurre, telle la lumière derrière la surface transparente d'une vitre. Martin Monestier (1999) mentionne l'invention en France à la fin du XIX siècle des premiers pièges à sucre, plus tard copiés en Allemagne et en Italie sous les noms d'« écrase-mouches à confiture " ou d'«écrase-mouches à miel». Les pièges sont composés de deux planchettes réunies par une charnière à ressort tel que sur les pièges à souris. Les deux faces internes sont garnies de sucre mouillée (ou de cancoillotte) qu'un manipulateur 
aux aguets actionne en libérant le ressort. Attirées par l'appât, les mouches sont alors écrasées entre les deux planchettes.

Revenons à l'insecte. Dans sa compréhension des signes qui l'entourent, la mouche est attirée par un aliment ou par une source de lumière : elle répondrait à une " tonalité de nutrition » ou à une "tonalité de parcours » selon Jakob von Uexküll (1934), avant de s'envoler à l'arrivée de la tapette ou de la planchette pour lui échapper en réponse à une "tonalité de fuite ou de défense ». Cette association d'un procédé d'attraction et d'un mode rapide de capture, pour reprendre la classification fonctionnelle de Monod, fait de ce type d'engins " éclairs » des pièges doublement mortels pour l'insecte. Ceuxci combinent les deux qualités du piégeage soulignées par Bahuchet et Pujol (1975) pour l'homme : le chasseur attend une réaction bien définie de la mouche, celle qui permet de l'attirer ; en même temps qu'il cherche à passer inaperçu de celle-ci.

La conception d'une tapette et de son mode d'action repose sur une compréhension humaine plus ou moins fine du comportement de l'insecte. La première tapette simple moderne brevetée aux États-Unis se devait d'être "élastique ", mais aussi «solide et durable" pour piéger d'un mouvement rapide mais précis (the whip-like swing) la

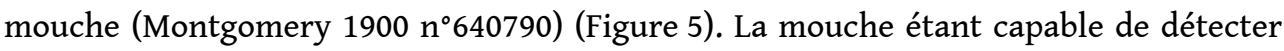
la pression de l'air (grâce aux poils microscopiques qui enveloppent son corps), la prise de la tapette à l'air est rapidement intégrée (voir pour une première synthèse sur les tapettes, Fraval 2008). Cyrus H. Porter fut le premier à mettre l'argument en avant dans la description du mode d'action du brevet qu'il déposa. Le tamis de son prototype de Fly-Killer est décrit comme « suffisamment ouvert pour qu'une fois le coup porté l'air passe au travers prévenant d'un déplacement d'air sur la mouche qui pourrait l'alerter avant l'impact » (Porter 1910 n $^{\circ} 975570$ ).

Autre modèle, mais un peu à part car sans descendance technique jusqu'ici, la tapette de William $\mathrm{H}$. Whitehead possède un maillage tubulaire qui entend prévenir que l'insecte « s'échappe avant le coup ou s'éloigne de celui-ci par le déplacement d'air qu'il produit» (Whitehead $1911 \mathrm{n}^{\circ}$ 984332) (Figure 5). D'autres prototypes prévoient de désorienter la mouche lors de l'envol par des tamis tournant sur un axe à $120^{\circ}$ (Pitts $1917 \mathrm{n}^{\circ} 1217212$ ) ou à $360^{\circ}$ (Sarantos $1934 \mathrm{n}^{\circ} 1984436$; Siggers $1972 \mathrm{n}^{\circ} 368402$ ). L'idée est d'étendre la distance d'action de la tapette en la prolongeant en cas d'obstacle, tel un coin de table ou une chaise, par une nouvelle rotation (Siggers $1972 \mathrm{n}^{\circ} 368402$ ) 
Figure 5 : Brevets de deux tapettes simples. Fly killer de Robert R. Montgomery (États-Unis, 1900, $\mathrm{n}^{\circ} 640790$ ) et fly killer de William H. Whitehead (États-Unis, 1911, $\mathrm{n}^{\circ} 984332$ )
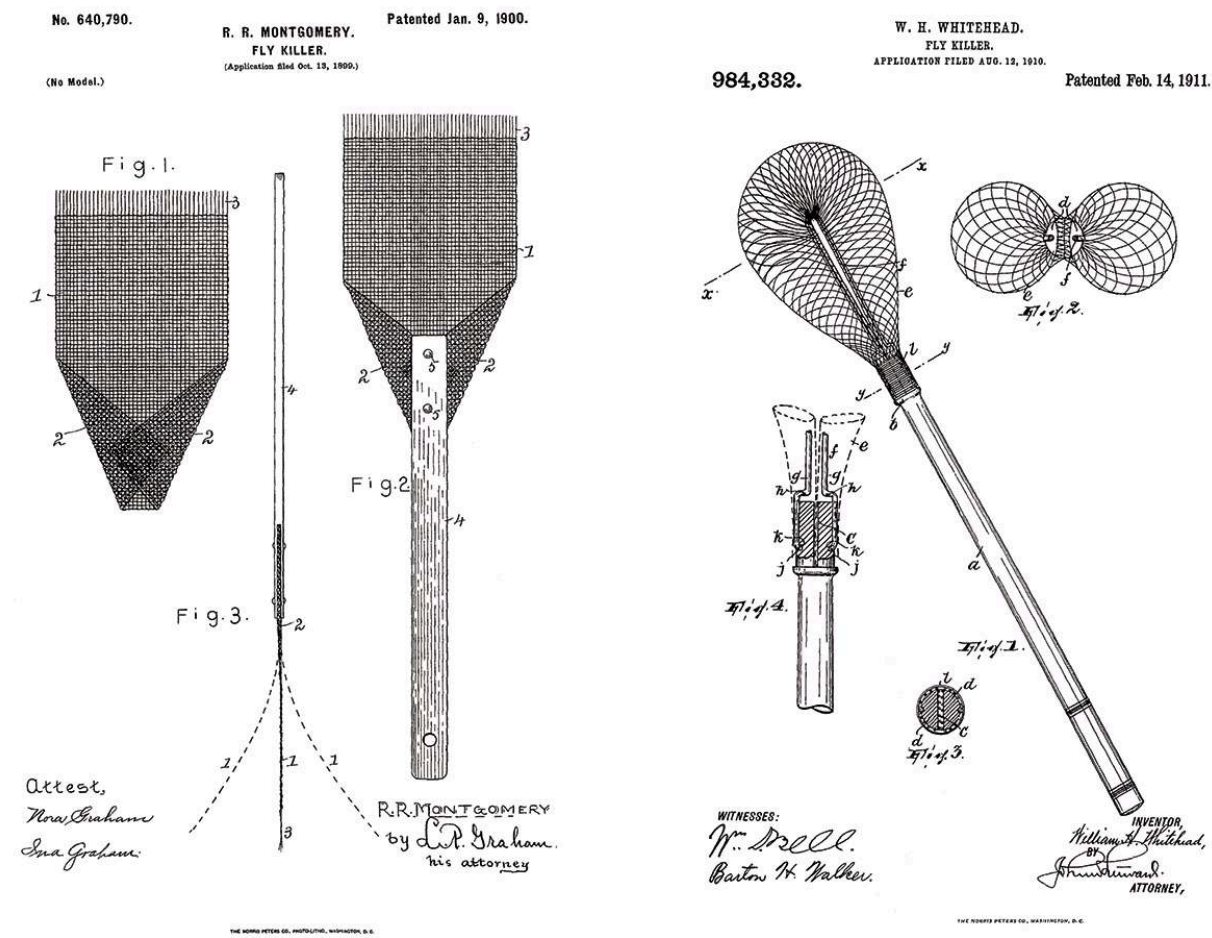

(C) www.freepatentsonline.com

Pour un meilleur usage des tapettes, les utilisateurs bénéficient désormais de conseils de spécialistes du comportement des insectes. Sollicité par la presse, l'entomologiste américain Michael Dickinson conseille de ne pas frapper directement la mouche mais de viser un peu plus loin devant elle pour anticiper son déplacement vers l'avant. L'approcher par derrière peut donner un léger avantage car si la mouche peut voir derrière elle sa vision n'est pas entièrement à $360^{\circ}$. Il conseille également l'emploi d'une tapette qui contraste peu avec le fond, c'est-à-dire d'une couleur aussi neutre que possible (lui-même utilise un simple journal roulé) ${ }^{4}$. Une autre biologiste, la britannique Kate Feller, suggère d'approcher la mouche très lentement pour désorienter sa perception visuelle très rapide ${ }^{5}$. De manière générale, la mouche étant un insecte à sang froid, il est suggéré d'essayer de l'attraper le matin ou le soir. 
Figure 6 : Quatre modèles de tapettes simples (États-Unis) ETB-US-NC-2019-06, ETB-USNC-2019-03, ETB-US-NC-2018-014, ETB-US-NC-2018-004
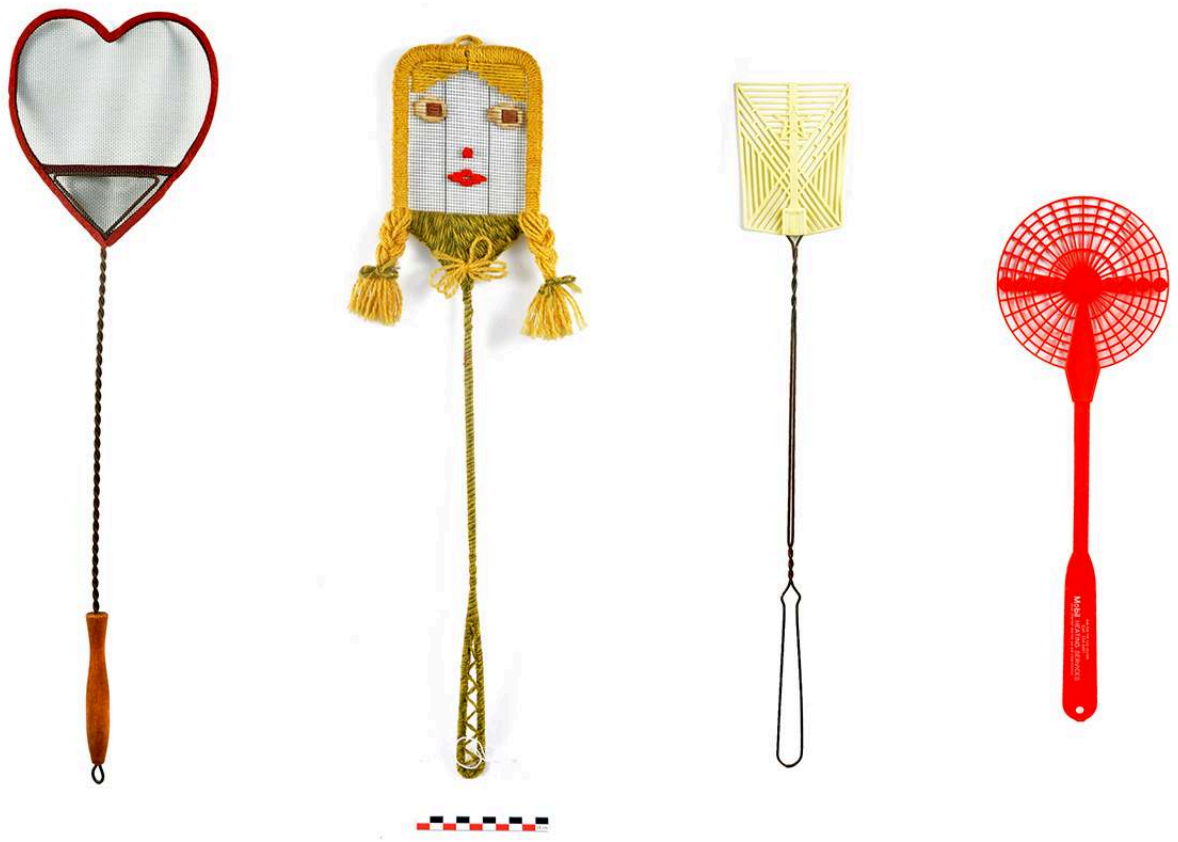

(C) M. Kourdourli/MNHN

19

- États-Unis ; années 1940

- Tapette en forme de cœur. Fer, tissu, bois

- Janvier 2019 ; collecteur Nicolas Césard

- Dimensions (cm) : 57 x 16

- No d'inventaire : ETB-US-NC-2019-06

20

- Tapette représentant une fillette. Fer, laine

- Février 2019 ; collecteur Nicolas Césard

- Dimensions (cm) : 56 x 11

- $\mathrm{N}^{\circ}$ d'inventaire : ETB-US-NC-2019-03

21 - États-Unis ; années 1960

- Tapette en fer et plastique

- Octobre 2018 ; collecteur Nicolas Césard

- Dimensions $(\mathrm{cm}): 53 \times 8,5$

- $\mathrm{N}^{\circ}$ d'inventaire : ETB-US-NC-2018-014

22 - États-Unis ; années 1960

- Tapette en plastique rouge de la compagnie Mobil Heating Services

- avril 2018 ; collecteur Nicolas Césard

- Dimensions (cm) : 41 x 13

- $\mathrm{N}^{\circ}$ d'inventaire : ETB-US-NC-2018-004 
Figure 7 : Quatre modèles de tapettes simples (France, Pérou, Indonésie) ETB-FR-NC-2019-11, ETBFR-NC-2019-010, ETB-PE-SB-2014-74, ETB-ID-NC-2019-01
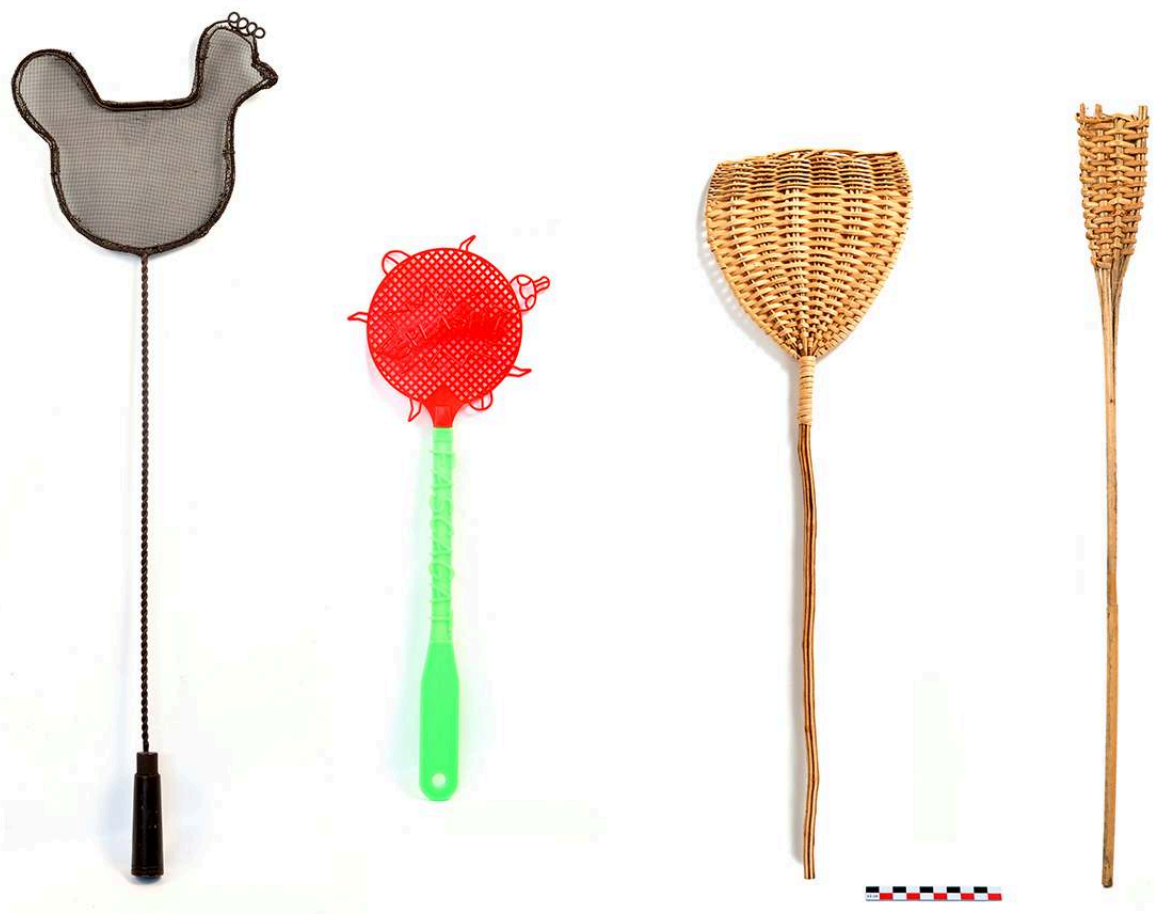

\section{(c) J. Tellier/M. Kourdourli/MNHN}

23

- France ; années 1950

- Tapette en forme de poule. Fer, bois

- Septembre 2019 ; collecteur Nicolas Césard

- Dimensions (cm) : 19,5 x 64

- Nº d'inventaire : ETB-FR-NC-2019-11

24 - France ; 2019

- Tapette en plastique Fasgacat fabriquée à Brusque (Midi-Pyrénées)

- Juillet 2019 ; collecteur Nicolas Césard

- Dimensions (cm) : 15,5 x 42

- $\mathrm{N}^{\circ}$ d'inventaire : ETB-FR-NC-2019-10

25 - Pérou; 2014

- Tapette en palmier Astrocaryum chambria, Iquitos (ethnie Yagua)

- Septembre 2014 ; collecteur Serge Bahuchet

- Dimensions (cm) : 16 x 55

- $\mathrm{N}^{\circ}$ d'inventaire : ETB-PE-SB-2014-74

26

- Indonésie ; 2019

- Tapette en rotin Calamus sp. (« frappe mouche », nampar lalat en dialecte local), île de Belitung

- Octobre 2019 ; collecteur Nicolas Césard

- Dimensions (cm) : 5,5 x 61

- $\mathrm{N}^{\circ}$ d'inventaire : ETB-ID-NC-2019-01 
À cette liste de pièges "éclairs", nous pourrions ajouter une paire de baguettes alimentaires, un ustensile proportionné à l'insecte et détourné en arme de chasse par le cinéma mais improbable car relevant d'une rapidité qui va au delà des capacités habituelles d'un chasseur de mouches (VIDEO 2).

Vidéo 2. Attraper les mouches avec des baguettes https://www.youtube.com/watch? v=wAuzCjipF00

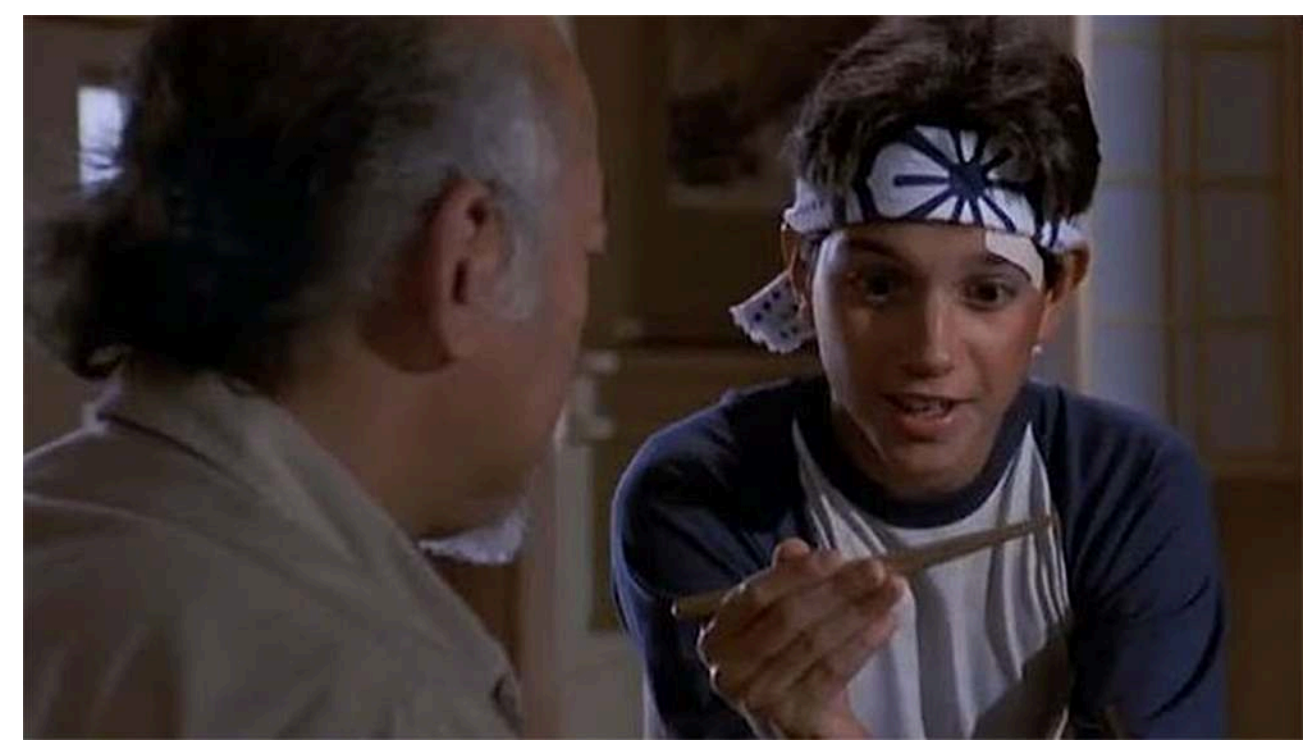

Extrait du film de John G. Avildsen, The Karate Kid (1984) avec Ralph Macchio et Pat Morita. Source Youtube Movieclips

\section{Pièges par « attraction » ou « appât »}

Une deuxième catégorie d'objets regroupe les pièges que nous nommerons pièges «par attraction » ou pièges "par appât », c'est-à-dire ceux qui attirent les insectes, via une source alimentaire pour les mouches et/ou de lumière pour d'autres insectes, pour les tuer ou les capturer vivants. Contrairement aux pièges " éclairs » qui les surprennent, ces derniers s'appuient sur le champ perceptif étendu des mouches pour les attirer.

Ici la mouche ne rentre pas en contact avec l'homme et se retrouve piégée avec ses congénères. L'avantage pour l'homme est qu'une fois le piège installé les insectes sont capturés sans qu'il intervienne. Deux types principaux seraient à distinguer selon leur issue, fatale ou non, pour les insectes. Les premiers, les pièges à appât disons " létaux ", disposent d'un attractif (toxique ou non) que la mouche consomme et d'une surface collante sur laquelle l'insecte est rapidement immobilisé, comme un fil trempé, de raphia, de ficelle ou de fer (Monestier 1999), plus récemment un ruban déroulant (Figures 8,15$)$ que l'on fixe au plafond ou un papier englué que l'on pose (Figure 1). Les mouches comme de nombreux autres insectes volants peuvent aussi être piégées par de la nourriture et/ou une source de lumière et être tuées au contact d'une grille dans laquelle passe un courant électrique, comme dans l'exemple de "L'électrocuteur " (Figure 9) qui combine les deux types d'appât, ou des pièges à rayonnement ultraviolet (également appelé « lumière noire ») utilisés dans les bâtiments agricoles. 
Figure 8 : Rubans attrape-mouches ETB-FR-NC-2019-09

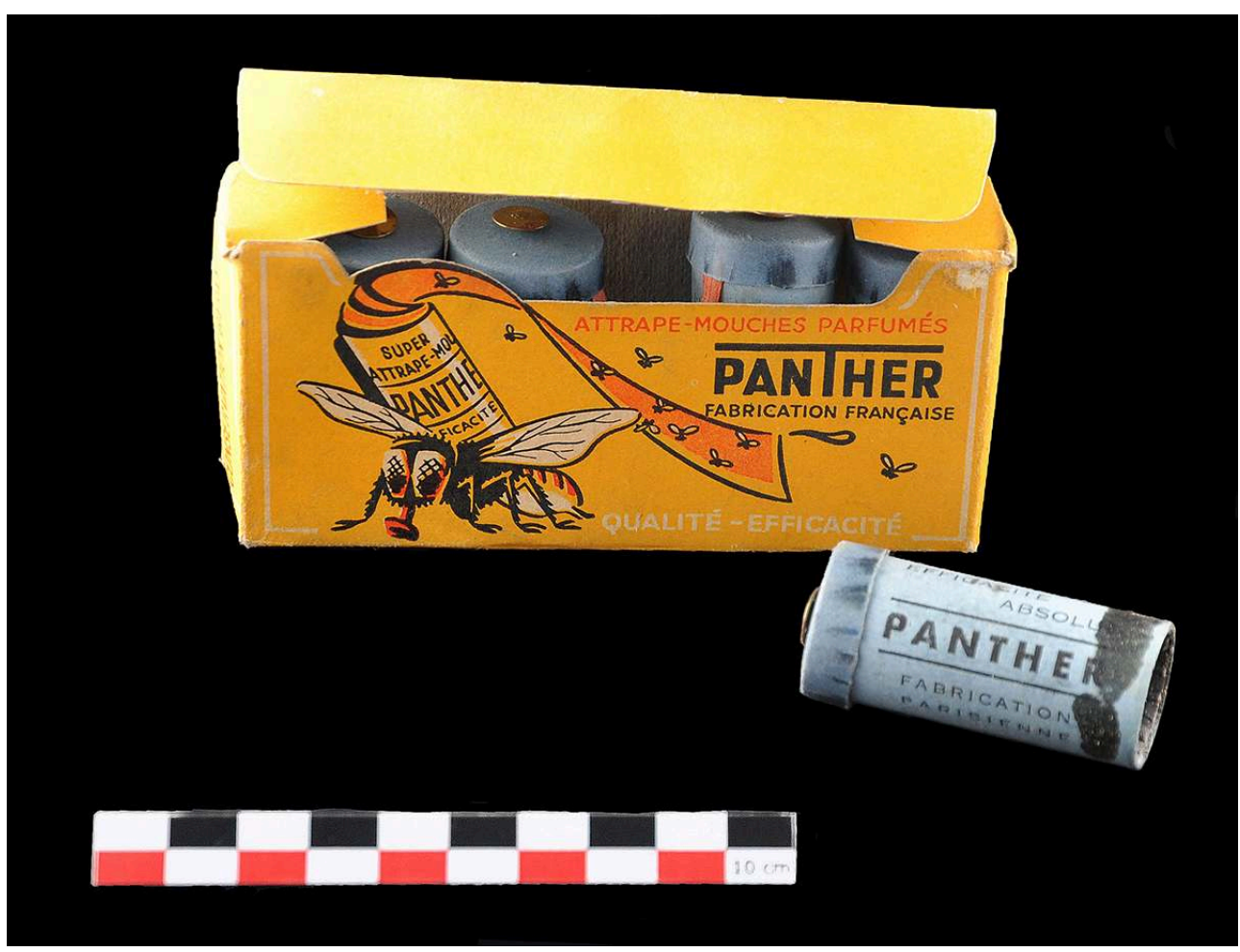

(c) M. Kourdourli/MNHN

- France ; années 1950

- Boîte de cinq rubans attrape-mouches parfumés de la marque Panther. La mouche est généralement attirée par une substance sucrée et non pas la nécessité de se poser. Elle est ensuite piégée par la colle ou la glu.

- Juin 2019 ; collecteur Nicolas Césard

- Dimensions (cm) : 12 × 5,5 x 2,2 (boîte)

- $\mathrm{N}^{\circ}$ d'inventaire : ETB-FR-NC-2019-09 


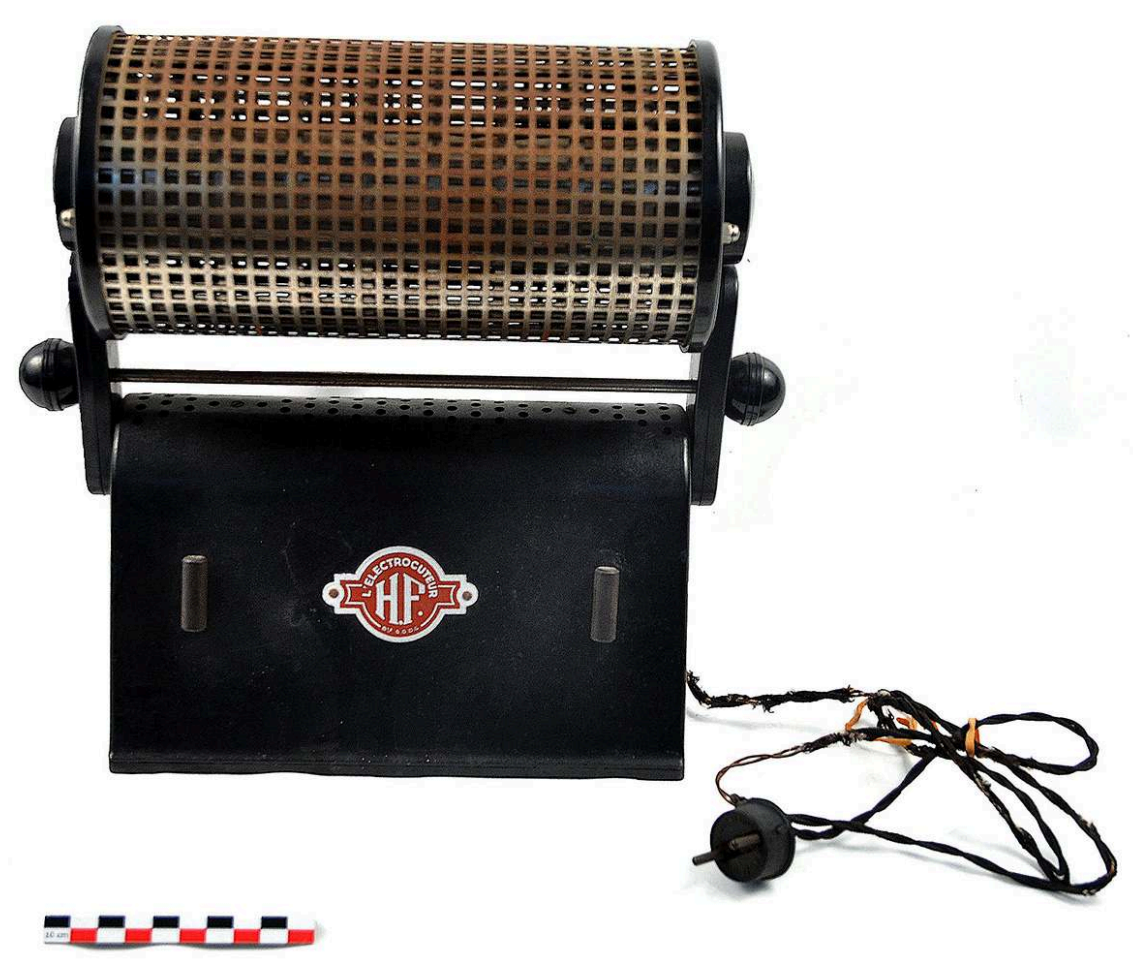

(c) M. Kourdourli/MNHN

- L'électrocuteur Homo-Flux, piège électrique à appât alimentaire et lumière (blanche). « Dès que les moustiques et autres insectes attirés par la lumière et par l'odeur d'un produit spécial se posent sur l'appareil, ils sont foudroyés ». "L'insecte établit luimême le contact qui l'électrocute ".

- Avril 2017 ; collecteur Nicolas Césard

- Dimensions (cm) : $31 \times 15 \times 30$

- $\mathrm{N}^{\circ}$ d'inventaire : ETB-FR-NC-2017-013

Les seconds, les pièges à appât que nous désignerons comme "captifs ", attirent les mouches mais les maintiennent enfermées, bien souvent jusqu'à épuisement, dessiccation ou noyade. La principale difficulté rencontrée par les utilisateurs est de pouvoir évacuer facilement les insectes (généralement morts) et de changer facilement l'appât souillé par les noyades. Les pièges les plus communs sont ceux en verre en forme de cloche ou de bouteille (dite « sans fond ») constituée d'une ouverture à la base entourée d'un appât alimentaire liquide (Figure 10) et les pièges grillagés dans lesquels les mouches appâtées entrent par une ouverture, souvent de la forme d'un entonnoir, sans possibilité d'en sortir (Figures 11, 12 et 15). 
Figure 10 : Gobe-mouches ETB-FR-NC-2017-01

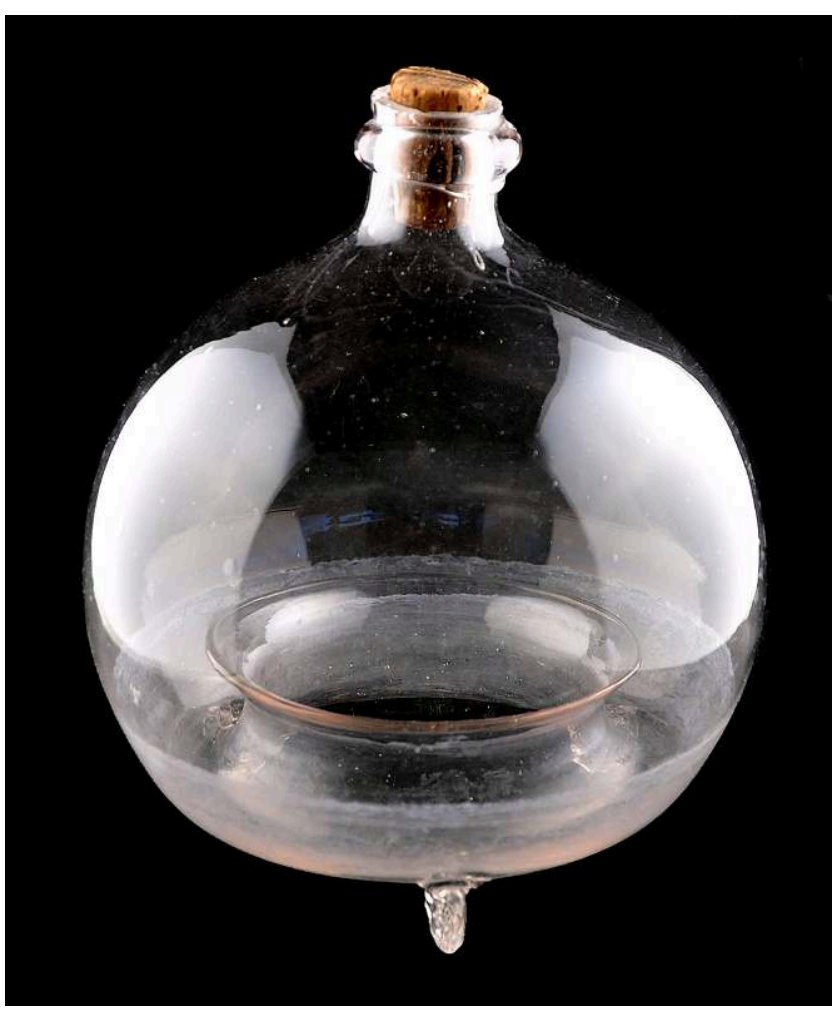

(c) M. Kourdourli/MNHN

33 - France ; années 1940

- Gobe-mouches ou bouteille "sans fond» en verre soufflé. Attirés par un appât alimentaire liquide généralement sucré, les mouches et autres insectes pénètrent par l'ouverture en dessous mais ne peuvent sortir et finissent noyés.

- Mai 2017 ; collecteur Nicolas Césard

- Dimensions (cm) 55 × 20

- $\mathrm{N}^{\circ}$ d'inventaire : ETB-FR-NC-2017-01 
Figure 11 : Piège à mouches grillagé ETB-FR-NC-2019-012

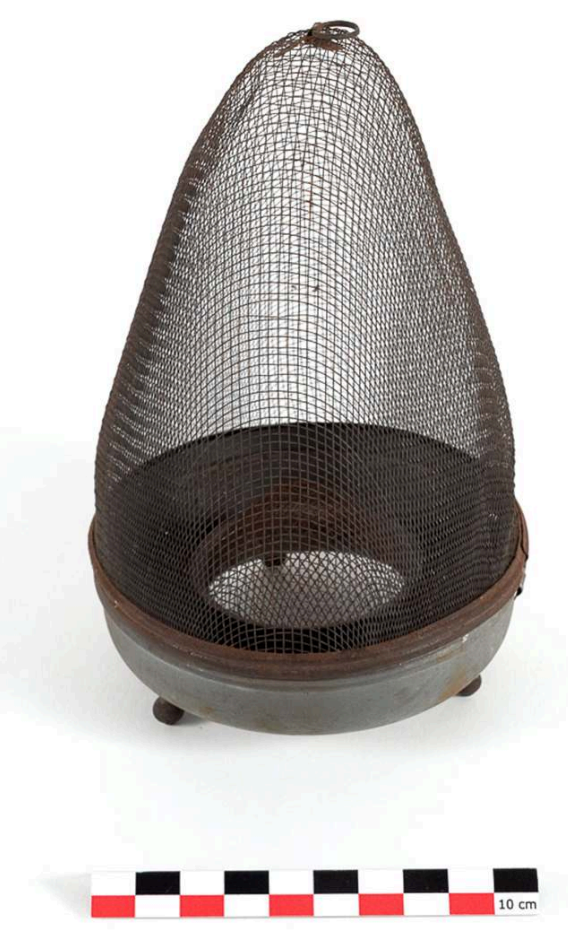

(c) J. Tellier

34 - France ; années 1920 ?

- Piège à mouches grillagé. Comme pour le gobe-mouches, l'appât est disposé dans la cage. Les mouches rentrent par l'entonnoir. Elles sont évacuées en dévissant la base du piège.

- Octobre 2019 ; collecteur Nicolas Césard

- Dimensions (cm) : $11 \times 17$

- No d'inventaire : ETB-FR-NC-2019-12 


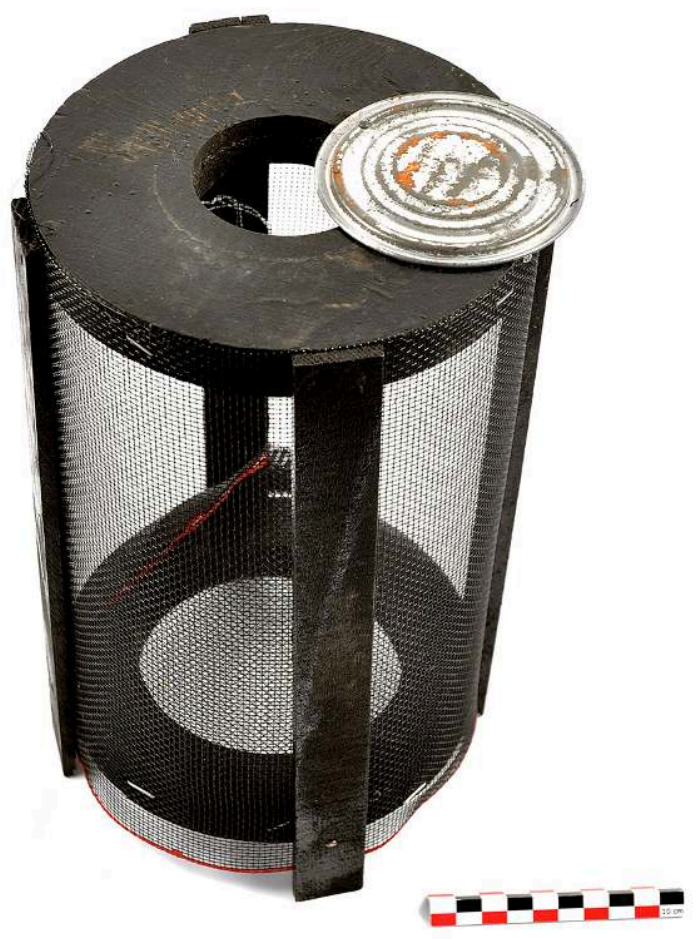

(c) M. Kourdourli/MNHN

35 - États-Unis ; années 1930

- Piège à mouches grillagé. L'appât est disposé sous l'entonnoir et les mouches sont évacuées par le dessus (ouvert à l'image) en retournant le piège. Piège "Sur-Katchem Fly trap » fabriqué par Olsen \& Sons, Kensett, Iowa, États-Unis.

- Juillet 2019 ; collecteur Nicolas Césard

- Dimensions (cm) : $28 \times 17$

- $\mathrm{N}^{\circ}$ d'inventaire : ETB-US-NC-2019-07

Enfin, dans les pièges mécaniques (Figures 13 et 15) ou électriques à appât (Figure 14), les mouches sont attirées par l'appât puis conduites ou «poussées » vers une cage ou un récipient fermé qui les isolent. En France, le piège à entonnoir mécanique de la marque Dubois, le bien nommé Mouchivore (Figure 15), connaîtra un certain succès au début des années 1900. Selon son catalogue de vente, il se compose "d'une boîte métallique circulaire dont le couvercle est animé d'un mouvement de rotation très lent qui lui est communiqué par un mécanisme intérieur pouvant fonctionner pendant dix heures. Ce plateau est garni de petites cavités dans lesquelles on place un peu d'eau sucrée ou de miel. Les mouches, attirées par cet appât, viennent se poser sur le plateau et sont ainsi entraînées en dessous d'une petite tour en toile métallique. Un petit racloir les force à rester dans cette tour où elles viennent s'emprisonner elles-mêmes ". (d'après Adeline Culas, écomusée de la Bresse bourguignonne, nº d'inv. EBB 99.24.56). 
Figure 13 : Piège mécanique à appât ETB-FR-NC-2018-008

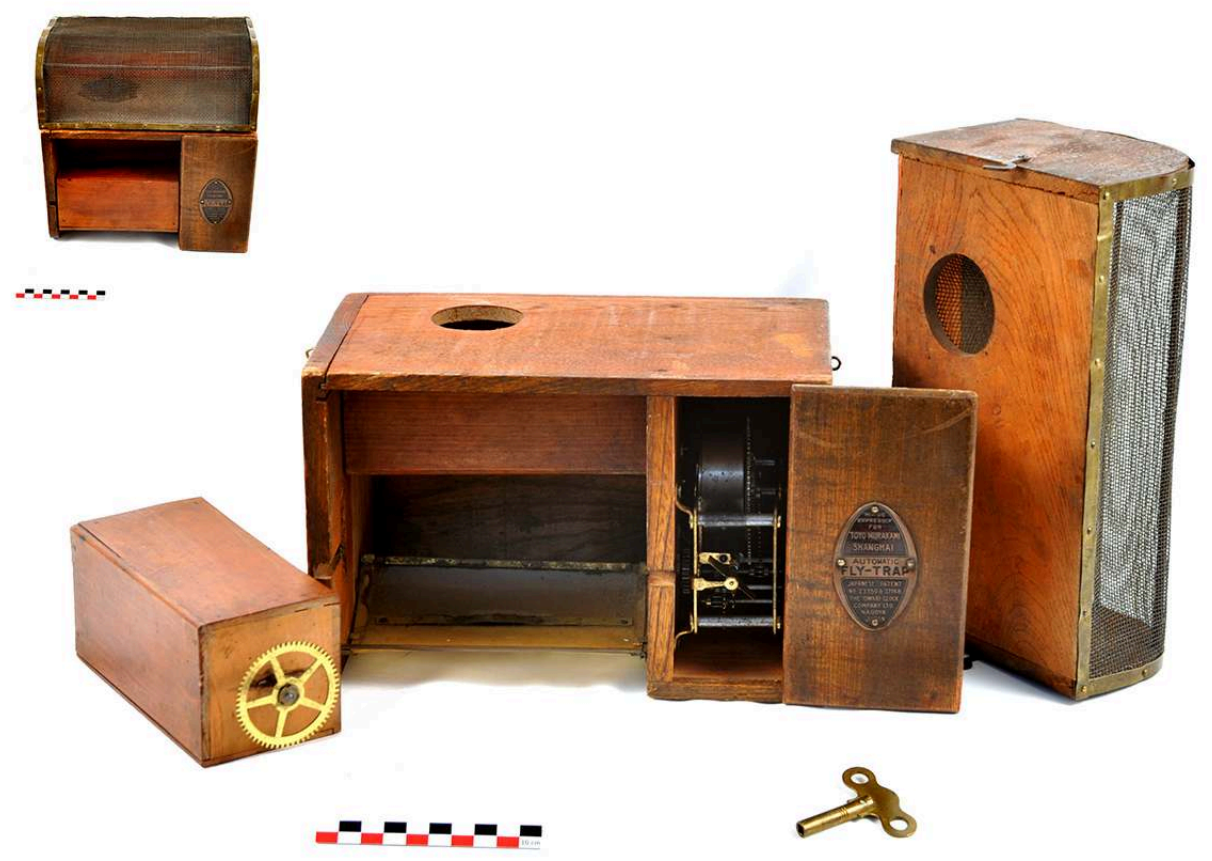

(c) M. Kourdourli/MNHN

- France ; année 1930

- Piège mécanique à appât (Automatic Fly trap) (les différents éléments sont séparés à l'image). Le piège contient les instructions suivantes : «Lorsque la machine doit être utilisée pour attraper des mouches, alimenter le rouleau avec du vinaigre ou un mélange de sucre et de liqueur. Pour les moustiques, n'utilisez que de l'alcool. Ces nuisances indésirables seront attirées par l'odeur et seront capturés dans la cage métallique prévue à cet effet. Lorsqu'elles sont attrapées et si vous souhaitez vous en débarrasser, tournez les crochets sur le côté de la machine et sortir la cage. Remontez l'horloge en douceur toutes les quatre ou cinq heures ». [Traduction des auteurs]. Fabriqué spécialement pour Toyo Murakami, Palace Hotel Building, Shanghai. Brevets japonais n²3359 \& 27748, mécanisme mécanique fabriqué par la compagnie d'horlogerie Owari située à Nagoya au Japon. Quant à Toyo Murakami, l'entreprise japonaise installée à Shanghai (Chine) fabriquait et exportait en Europe et aux ÉtatsUnis différentes curiosités de facture principalement chinoise. Le piège fut commercialisé en France sous le nom de Muscamor dès les années 1890 (produit par Cizeron, 50 rue d'Alger à Toulon).

- Février 2018 ; collecteur Nicolas Césard

- Dimensions (cm) $24 \times 24 \times 14$

- No d'inventaire : ETB-FR-NC-2018-008 
Figure 14. Piège électrique à appât ETB-CH-NC-2019-01

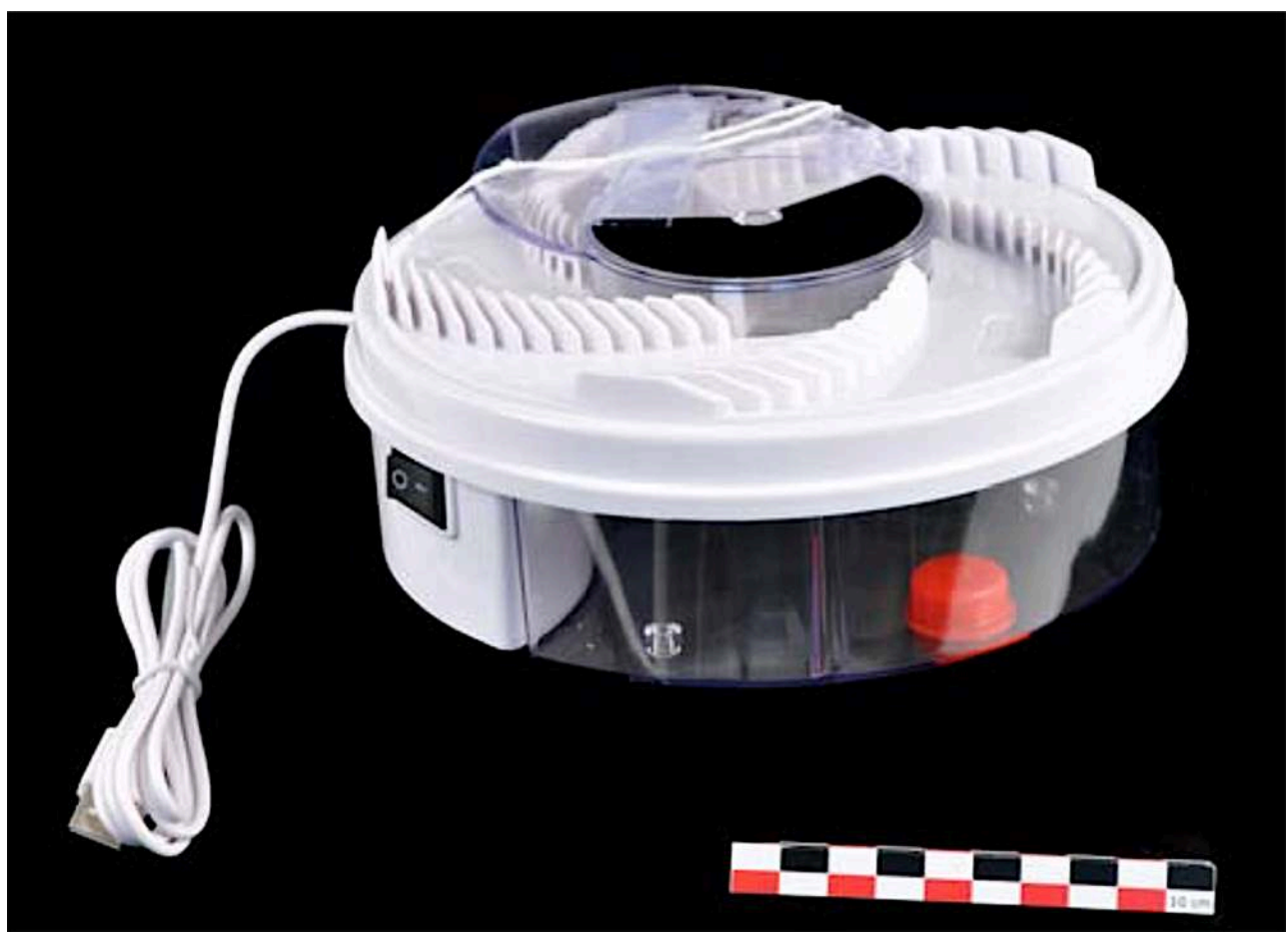

(c) M. Kourdourli/MNHN

- Chine ; 2019

- Piège électrique à appât (Automatic Flytrap). « Home-friendly ways to get rid of houseflies ». «Déposez quelques gouttes de miel dans le couloir de piégeage et branchez l'alimentation électrique, l'appareil commence à fonctionner lorsque les plaques tournent. Les mouches consomment les appâts et sont repoussées vers la boîte pendant que les plaques tournent. Lorsque un nombre suffisant de mouches est capturé, débranchez l'alimentation et enlevez la boîte, ou retirez le bouchon de sortie pour évacuer les mouches et les mettre à la poubelle ». [Traduction des auteurs]. Pour une vidéo de l'objet fonctionnant, voir https://www.youtube.com/watch?v=lEsPv1h5kWo

- Dimensions (cm) : $21 \times 7,5 \times 20,5$

- Février 2019 ; collecteur Nicolas Césard

- $\mathrm{N}^{\circ}$ d'inventaire : ETB-CH-NC-2019-01 
Figure 15 : Publicité pièges à appât ETB-FR-NC-2019-013

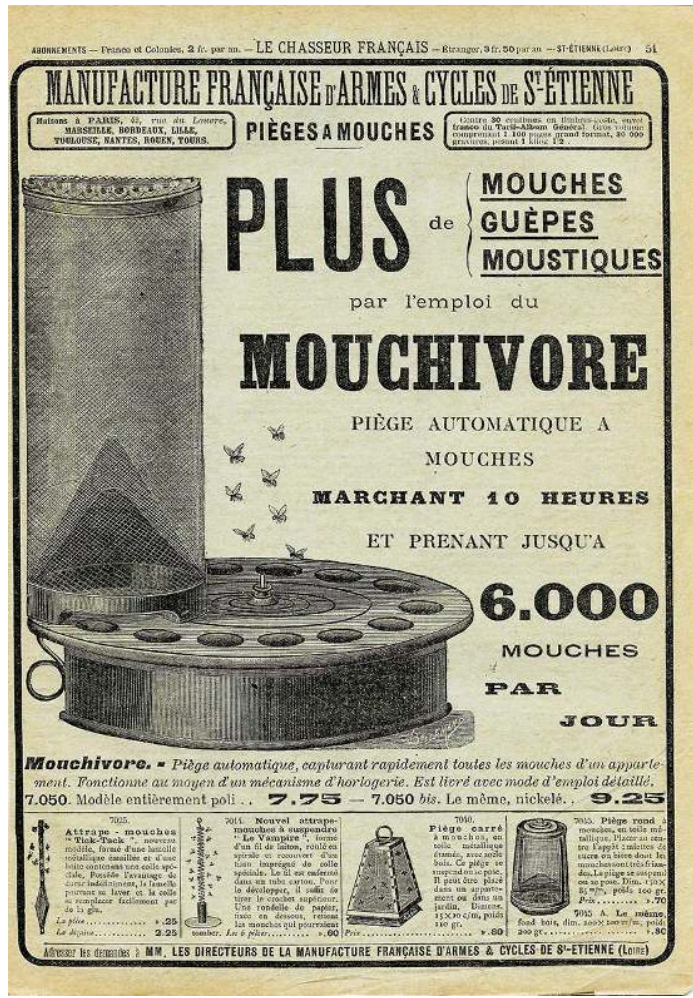

(c) N. Césard/MNHN

39 - France ; aout 1911

- Publicité du magazine Le Chasseur Français (Saint Etienne) pour le Mouchivore, piège automatique à mouches, et pour d'autres pièges à appât.

- Novembre 2019 ; collecteur Nicolas Césard

- Dimensions $(\mathrm{cm}): 17$ x 25

- Nº d'inventaire : ETB-FR-NC-2019-13

\section{Genèse des pièges à mouches}

Les modes d'action des différents objets présentés répondent à ceux de l'insecte comme aux contraintes techniques pour les piéger mais que disent-ils de nos relations aux insectes? Les attitudes des hommes, leurs choix comme leurs usages de la technique, s'appuient sur des représentations culturelles, écologiques ou historiques dont découlent les relations au vivant. Un chasse-mouche repousse les mouches mais il peut être utilisé par conviction religieuse (le balai ou le cache-bouche des mendiants jaïns d'Inde pour éviter d'écraser ou d'ingérer un insecte) ou pour ce qu'il symbolise (en Afrique, un objet pour chasser les «mouches » responsables de la filariose mais aussi un objet de pouvoir pour éloigner les esprits invisibles). Or, si dans nos exemples un piège à mouches sert principalement à éloigner, tuer ou capturer, les usages (et les mésusages) qui en sont faits donnent peu d'informations sur les intentions initiales de leur créateur. La finalité d'un objet pouvant varier, cette partie propose de revenir sur la genèse de certains objets, c'est-à-dire sur les raisons (notamment morales) qui ont motivé leur création. Suivant à nouveau les conseils de George Simondon (1958), nous 
partirons « des critères de la genèse » pour "définir l'individualité et la spécificité de l'objet».

Selon Simondon, un objet technique appartient à la fois à un milieu "géographique " (au sens large, c'est-à-dire de milieu "naturel ») et à un milieu "technique », deux mondes entre lesquels l'activité technique consiste à trouver un "compromis». Or la " concrétisation " d'un objet tel un piège à mouches n'est pas tant fonction de son adaptation à un milieu ou à un autre qu'elle n'incombe à la nature de la relation entre ces deux milieux (d'après Duperrex 2019). Les inventions et les innovations successives intègrent des préoccupations biologiques, techniques, mais aussi économiques, esthétiques, voire éthiques, qui nécessitent un détour par l'histoire des idées et une description davantage socio-technique de leur émergence. Pour comprendre la popularité de la tapette à mouches moderne et la variété des modèles, il est nécessaire de revenir brièvement sur l'histoire des États-Unis.

\section{La tapette américaine, instrument de santé publique}

42 L'usage des tapettes se développe au début du $x^{e}$ siècle aux États-Unis et accompagne un changement de perception de l'insecte. À la sortie de la guerre civile, en 1865, les citoyens américains redoublent d'efforts pour moderniser leurs villes et offrir à leurs habitants de meilleures conditions sanitaires. Des systèmes d'eau potable et d'évacuation sont installés et les routes et trottoirs sont améliorés et uniformément pavés $^{7}$. Parallèlement, et cela malgré des preuves scientifiques limitées, les réformateurs de la santé publique tiennent pour responsable de la propagation des maladies un insecte commun et jusqu'ici inoffensif, la mouche domestique (Musca domestica). L'insecte domiciliaire devient vecteur de différentes épidémies dont la fièvre typhoïde, la diarrhée infantile et la tuberculose. Durant les trois décennies suivantes, les services sanitaires américains vont s'efforcer d'avertir le public des dangers de l'insecte : des brochures sont publiées et diffusées et des concours sont organisés pour encourager la destruction des mouches et palier les facteurs environnementaux supposés responsables de leur prolifération.

Selon Naomi Rogers, cette "diabolisation» des mouches témoigne du passage au tournant du siècle de la pratique de la science sanitaire à la nouvelle santé publique (Naomi Rogers 1989 citée par Minnett et Poutanen 2007). En effet, si le public associe volontiers la présence de saletés et de déchets aux maladies, il est plus difficile de "montrer» aux gens que les germes microscopiques sont responsables de la propagation des infections. Afin de faciliter la compréhension et l'acceptation de la théorie microbienne des maladies, les scientifiques ont alors l'idée de modifier la perception du public, faisant passer les mouches d'insectes familiers à des «bactéries sur pattes» (germs with legs). Les comportements de la mouche expliquent la présence de microbes invisibles et leur propagation, en même temps qu'ils appellent les citoyens avertis à l'action personnelle et à la surveillance, tels la présence d'écrans protecteurs aux fenêtres et l'usage de tapettes pour les détruire (id.).

La problématique de la propagation des maladies par la mouche n'apparait toutefois pas tout de suite comme une motivation et un objectif des inventeurs de tapettes modernes. Les tapettes trouvent d'abord progressivement leur place dans l'équipement des ménages américains. L'invention de Robert R. Montgomery, le Fly-Killer, fut présentée dans le magazine féminin Ladies Home Journal de 1901 comme un objet qui 
«tue sans écraser $»^{8}$, sans que le lien avec la diffusion des maladies soit évoqué. Outre leur simplicité, leur durabilité et leur coût, celles de Irvin H. Ames, de Porter (1910 $\mathrm{n}^{\circ}$ 975570) et de Charles W. Merrifield (1912 $\left.\mathrm{n}^{\circ} 1030004\right)$ sont présentées comme des dispositifs «qui n'abiment ou ne souillent pas les meubles, le papier peint, les rideaux ou tous les autres tissus sur lesquels la mouche peut être frappée» (Ames 1902 $\mathrm{n}^{\circ}$ 695189). À partir de 1913, les brevets vantent encore leur petite taille par rapport aux autres pièges ménagers existants mais l'argument sanitaire devient plus explicite (Allis \& Wiens $1913 \mathrm{n}^{\circ} 1055998$ ).

Figure scientifique et politique, le responsable de l'entomologie dans le gouvernement fédéral américain, Leland 0 . Howard, est un des premiers à mettre en avant les avantages de l'entomologie appliquée à la santé publique (Russell 1999). Usant de ce qu'il nomme « une approche psychologique ", il n'hésite pas à employer les métaphores guerrières pour convaincre. En 1908, il propose - sans succès - de changer le nom de la mouche domestique par celui de mouche typhoïde plus à même de susciter l'appréhension de ses concitoyens et donc la volonté de la combattre. L'idée fait toutefois son chemin et suscite une couverture médiatique importante en 1910 lors de la campagne nationale Swat the Fly («Frapper la mouche»). L'Association Civique Américain (ACA) prépare des présentations dans la presse, des brochures (Figure 16), des posters et même un film. Howard demande notamment que les enfants, plus influençables selon lui que les adultes, soient concernés par la campagne (id.).

Figure 16 : Brochure de prévention ETB-US-NC-2019-08

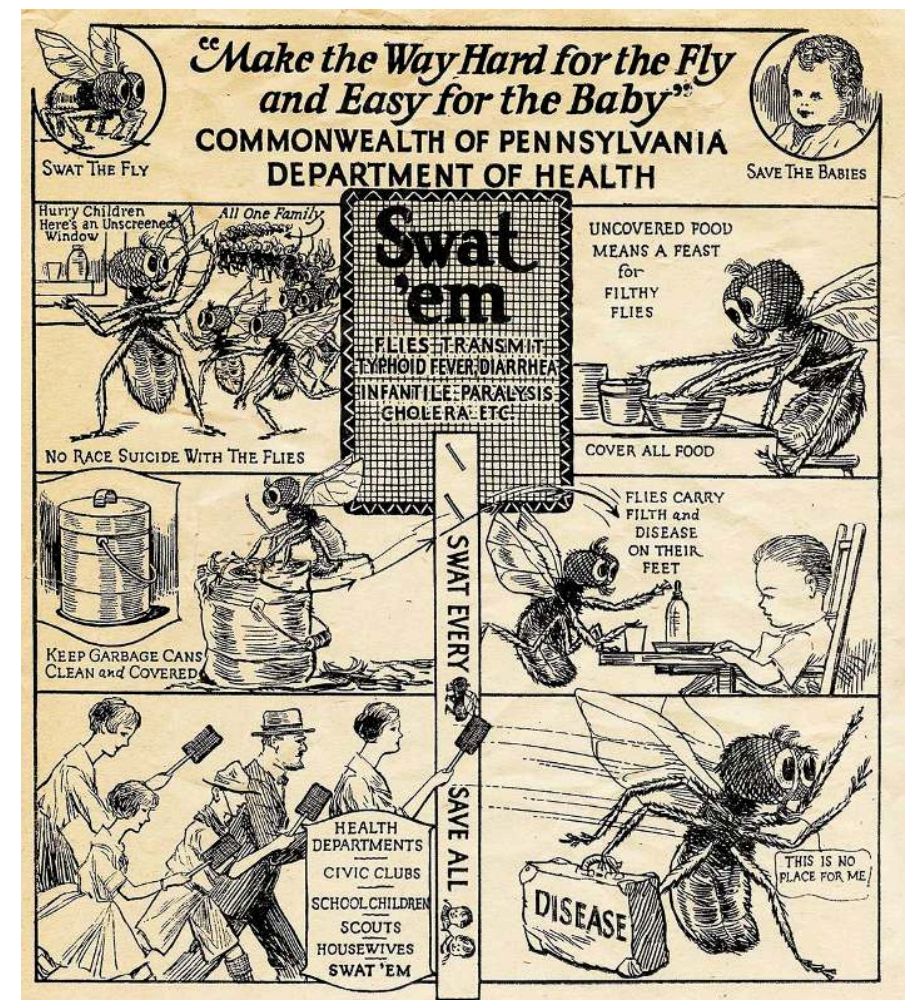

(C) N. CÉSARD/MNHN

46 - États-Unis ; années 1930

- Brochure de prévention "Make the Way Hard for the Fly and Easy for the Baby. Swat'em ». Département de la santé de Pennsylvanie. En réalité, la campagne Swat the fly 
commence quelques années plus tôt, en 1905, à l'initiative du secrétaire du Conseil de la santé de l'État du Kansas, le docteur Samuel J. Crumbine9. Il est le premier à faire paraître une brochure de prévention du même nom après avoir entendu un fan de base-ball s'exclamer lors d'un match : « Avez-vous vu comment il l'a mouché (swat that fly)!", une fois la balle propulsée hors du terrain. Peu après Frank H. Rose et les scouts de la ville de Weir, toujours dans le Kansas, installèrent des rideaux de fenêtres antimouches pour que les habitants se protègent et utilisèrent les restes de treillis en les attachant à des manches créant ce qu'ils appelèrent des fly bats ("battes à mouche »). Rose montra ces nouveaux engins à Crumbine qui leur donna le nom de fly swatters, un terme qui désigne encore aujourd'hui aux États-Unis, et au delà, les tapettes à mouches. - Juillet 2019 ; collecteur Nicolas Césard

- Dimensions (cm) : 25 x 23

- $\mathrm{N}^{\circ}$ d'inventaire : ETB-US-NC-2019-08

Les nouveau-nés en particulier sont désignés comme les premières victimes des mouches. Lors des campagnes de sensibilisation, des pièges et des tapettes sont distribués aux adultes et aux enfants en âge de chasser (Figures 17, 18 et 19). À Westminster dans l'État du Maryland, ces derniers reçoivent jusqu'à 10 cents par centaine de mouches tuées. L'année 1914, la ville dépense 159 dollars et distribue 1500 tickets de cinéma en contrepartie des seaux de mouches collectés ${ }^{10}$.

Figure 17 : Piège à mouches grillagé ETB-US-NC-2019-002
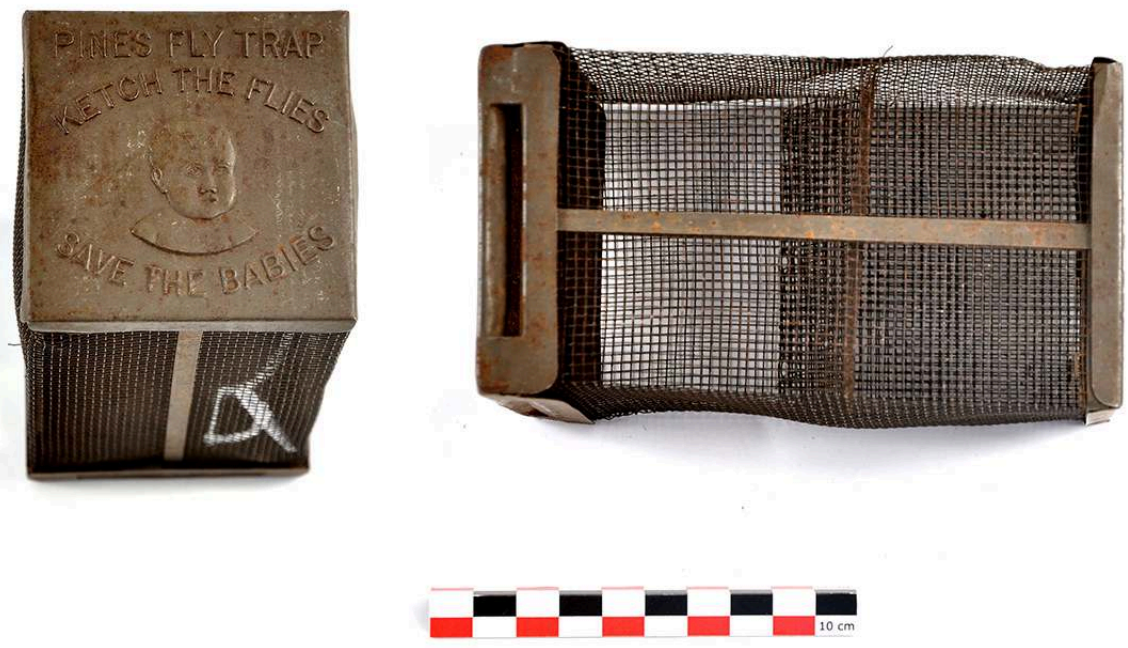

(c) M. Kourdourli/MNHN

48 - États-Unis

- Piège à mouches grillagé Pines Fly Trap avec inscription « Ketch the flies, save the babies

» d'un côté et « Appâter avec du sucre et du vinaigre » de l'autre.

- Février 2019 ; collecteur Nicolas Césard

- Dimensions (cm) 13,5 x 7,5

- $\mathrm{N}^{\circ}$ d'inventaire : ETB-EU-NC-2019-002 
Figure 18 : Tapette à mouches publicitaire ETB-US-NC-2018-013

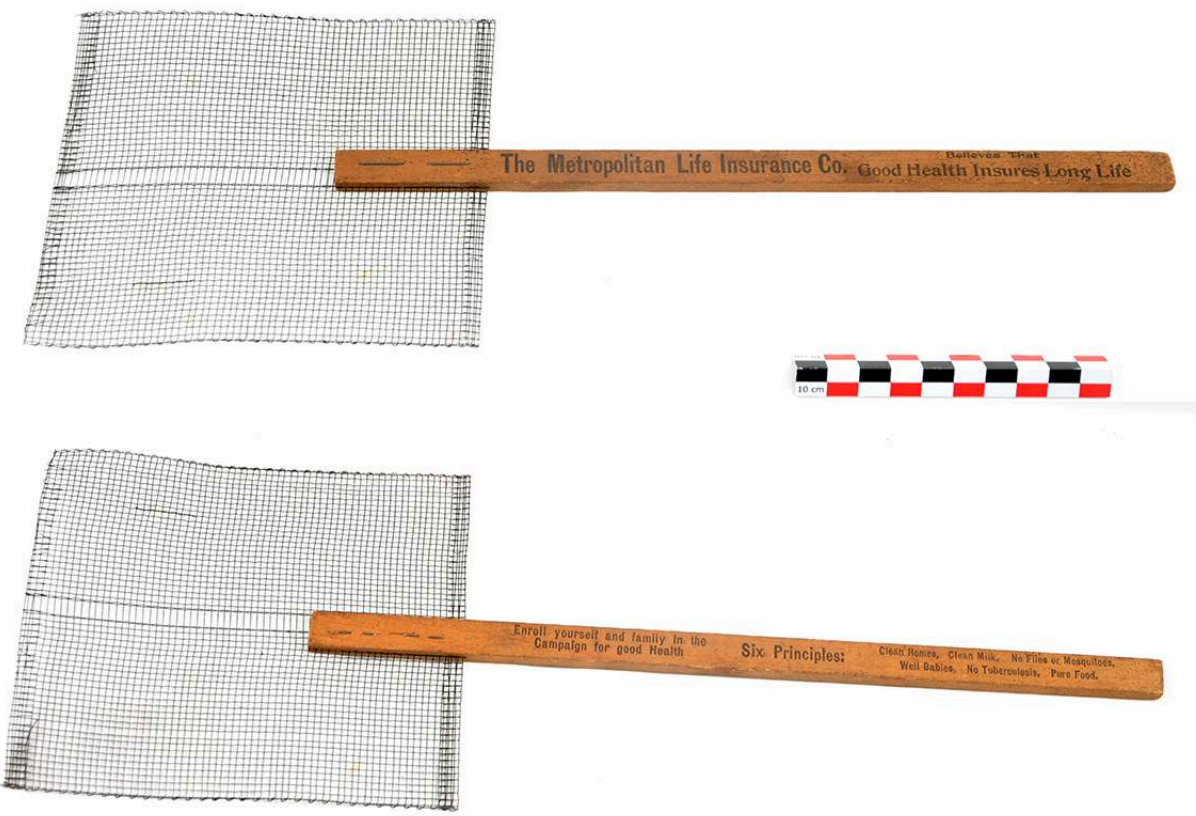

(c) M. Kourdourli/MNHN

49 - États-Unis ; années 1920

- Tapette à mouches publicitaire avec au recto: «La compagnie Metropolitan Life Insure croit qu'une bonne santé assure une longue vie». Au verso : «Inscrivez-vous, vous et votre famille, à la campagne pour une bonne santé. Six principes : Des maisons propres, du lait propre, pas de mouches ou de moustiques, des bébés bien portants, pas de tuberculose, de la nourriture pure ». [Traduction des auteurs]

- Métal et bois

- Décembre 2018 ; collecteur Nicolas Césard

- Dimensions (cm) : $37 \times 12$

- $\mathrm{N}^{\circ}$ d'inventaire : ETB-EU-NC-2018-013 
Figure 19 : Tapette à mouches publicitaire ETB-US-NC-2018-011
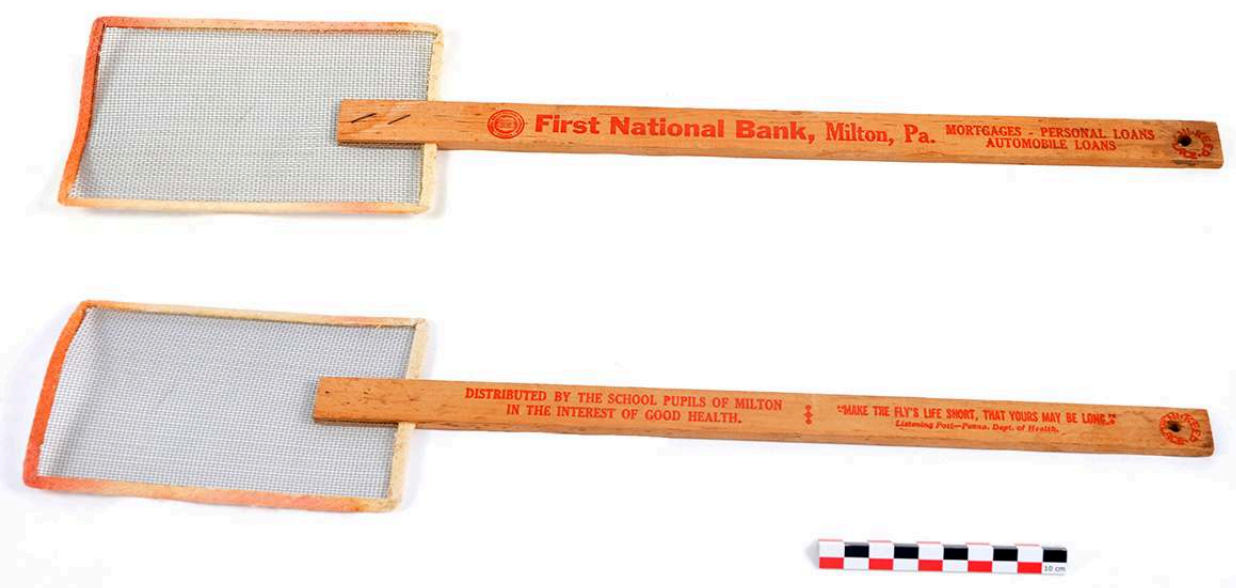

(c) M. Kourdourli/MNHN

50 - États-Unis ; années 1920

- Tapette à mouches publicitaire avec au recto: "First National Bank, Milton, Pa. Hypothèques, prêts personnels. Prêts automobiles». Au verso: "Distribué par les élèves de Milton dans l'intérêt de la santé. « Rends la vie de la mouche courte, afin que la tienne soit longue ». Poste d'écoute - Ministère de la Santé de Pennsylvanie». [Traduction des auteurs]

- Métal et bois

- Octobre 2018 ; collecteur Nicolas Césard

- Dimensions (cm) : $49 \times 10$

- $\mathrm{N}^{\circ}$ d'inventaire : ETB-US-NC-2018-011

51 Progressivement le terme de fly-swatter s'impose dans le nom des brevets. En 1918, la tapette est nommée alternativement fly-killer ou fly-swatter mais l'invention garde encore le nom de fly-killer (Hall \& Fields $1918 n^{\circ} 1286894$ ) qui disparaît définitivement à partir de 1920 pour prendre l'appellation de fly-swatter (Graham 1920 n¹344943) (Figure 20). 
Figure 20 : Brevets de deux tapettes simples
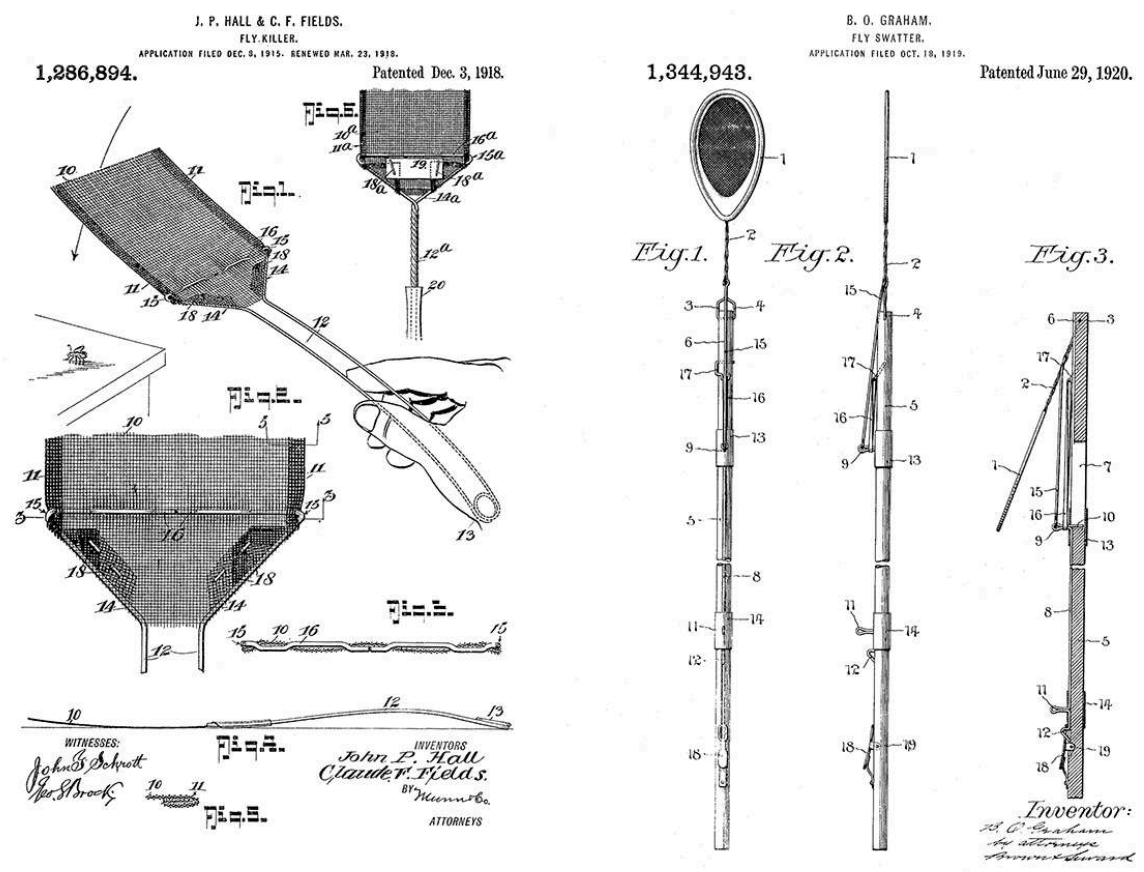

Fly-killer de John Porter Hall et Claude F. Fields (États-Unis, 1918, n¹286894) et fly-swatter de Bert O. Graham (États-Unis, 1920, n¹344943).

(C) www.freepatentsonline.com

En 1921, le brevet du premier fly-swatter sous forme de pistolet est déposé. Il est décrit comme un jouet « pour susciter et maintenir l'intérêt des enfants dans les campagnes

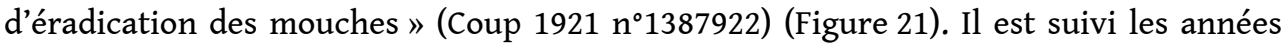
suivantes de modèles plus sophistiqués à la fois instruments de chasse et moyens d'amusement (Kennedy \& Saiger Fly shooter 1922 n¹457674; Blake Fly swatting device $1923 n^{\circ} 1468373$ ) (Figure 21). 
Figure 21 : Brevets de deux tapettes simples
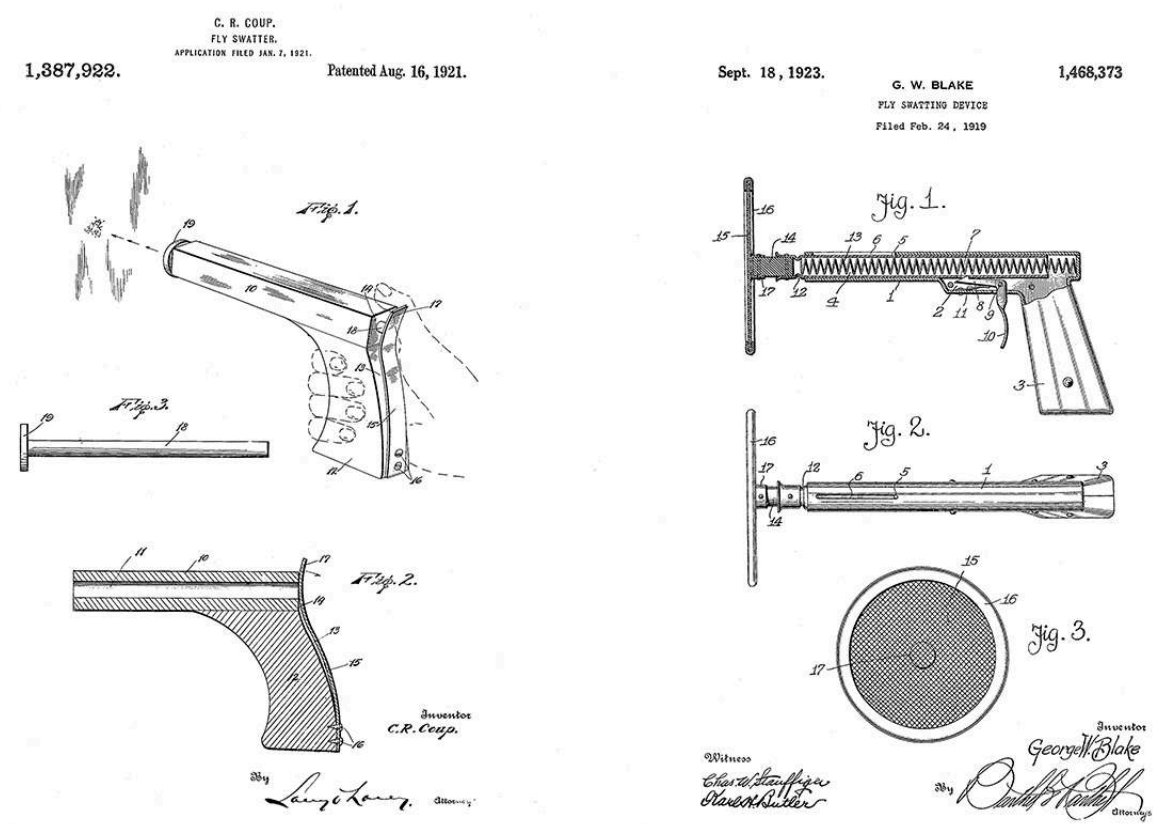

Fly swatter de Charles R. Coup (États-Unis, 1921, n¹387922 et Fly swatting device de George W. Blake (États-Unis, 1923, n¹468373)

(c) www.freepatentsonline.com

$\mathrm{Au}$ fil des décennies, le côté ludique prend le pas sur les préoccupations sanitaires. Le modèle récent de la famille Belokin (Belokin et al. 2006 USD527774S, Figure 22) connaît un certain succès commercial auprès des chasseurs de mouches. Puis, alors que «les autres engins sont connus pour tirer un projectile avec pour objectif de toucher ou éliminer les insectes", Bruce Nordstog développe en 2011 un prototype dont le projectile est conçu explicitement non pas pour écraser l'insecte mais pour le capturer (sans préciser vivant ou mort) (Nordstog 2011 US7861698B2, Figure 23). L'invention cite les 35 brevets précédents qui l'ont inspiré (le premier datant de 1919, le dernier de 2006) et les divise en trois groupes : un modèle initial avec un projectile composé d'un ressort pour donner de la puissance (Belokin $1980 \mathrm{n}^{\circ}$ 4195615), un second groupe d'inventions autour du projectile et de son système de blocage, et un dernier sur les engins et les mécanismes de capture de l'insecte. 
Figure 22 : Tapette jouet et brevet du prototype ETB-UK-NC-2019-03

|||||||||||||||||||||||||||||||||||||||||

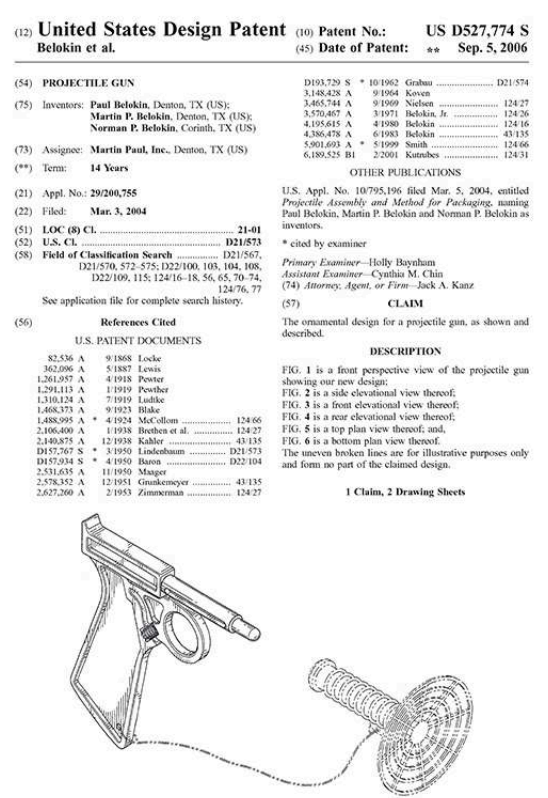

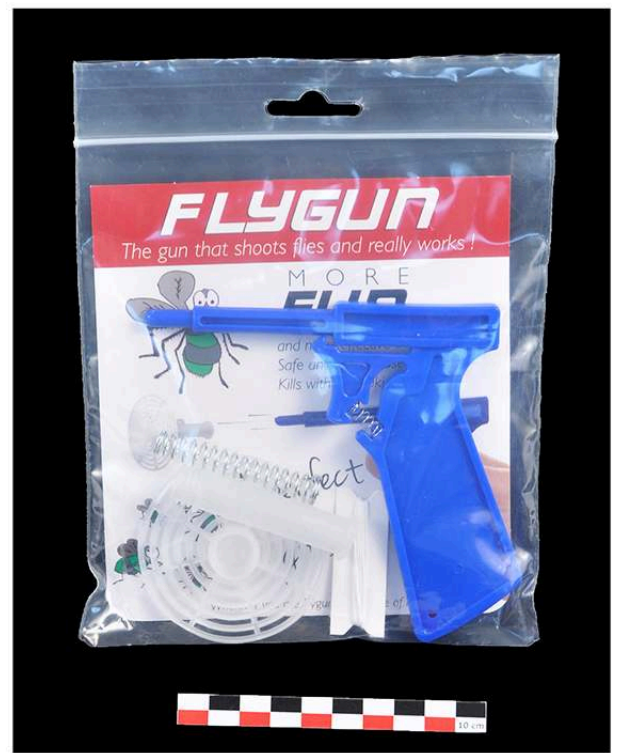

(c) www.freepatentsonline.com (CM. Kourdourli/MNHN

54

- Projectile gun de Paul Belokin Martin P. Belokin et Norman P. Belokin (États-Unis, 2006, USD527774S) (source: www.freepatentsonline.com) commercialisé sous le nom de Flygun ETB-UK-NC-2019-03.

- Juillet 2019 ; collecteur Nicolas Césard

- Dimensions (cm) $13 \times 7$

- Nº d'inventaire : ETB-UK-NC-2019-03 
Figure 23 : Brevet d'une tapette conçue pour capturer les insectes

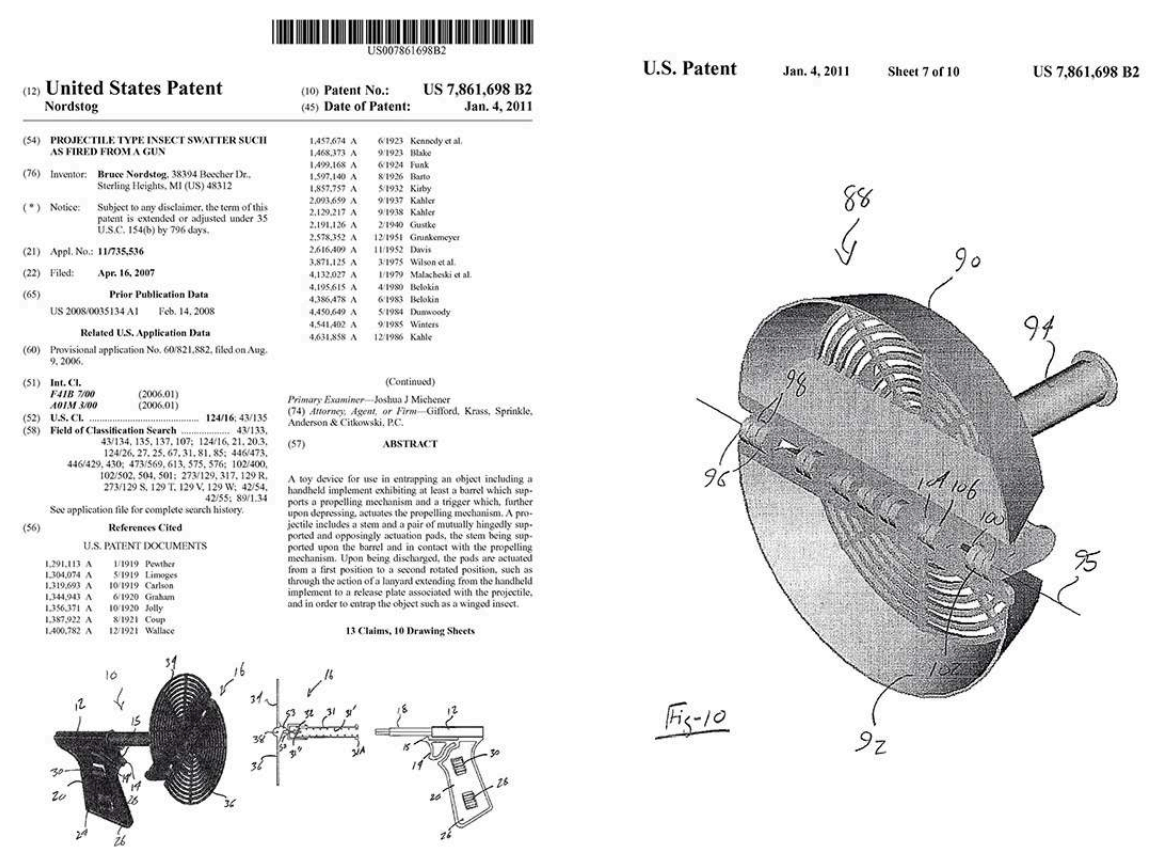

PROJECTILE TYPE INSECT SWATTER SUCH AS FIRED FROM A gUN DE BRUCE NORDSTOG (ÉTATS-UNIS, 2011, N ${ }^{\circ}$ S7861698B2)

(C) WWW.FREEPATENTSONLINE.COM

\section{Du piège létal au piège éthique}

Si plusieurs pièges "par attraction » ou "par appât » sont conçus pour capturer les mouches dans la perspective de les tuer, d'autres ne précisent pas leurs intentions ou sont créés pour capturer les insectes puis pour les libérer. Les mécanismes de ces pièges "captifs " pour reprendre notre distinction prennent en compte un comportement attendu de l'insecte mais leur intention est différente. Seul un retour sur leurs objectifs, instructions ou modes d'emploi, permet de comprendre leur fonction initiale, leur spécificité. Les premiers fly trap ou pièges à appât captifs sont pensés " pour enfermer [les mouches] et permettre de tuer facilement les insectes quand un nombre suffisant est capturé »(Wallace $1912 \mathrm{n}^{\circ}$ 1017027) (Figure 24, voir également Figure 13), en particulier dans les lieux communs comme les hôpitaux ou les hôtels «où il est souhaitable d'attraper les mouches vivantes pour les détruire par la suite» (Yokosawa \& Miyazaki $\left.1923 \mathrm{n}^{\circ} 1471086\right)$. Quant à la finalité du premier « aspirateur » à insectes, un piège mécanique à piston appelé Flycatcher, il s'arrête à la capture et ne tranche pas

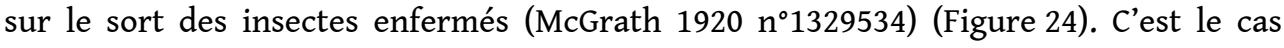
également de son successeur motorisé (Catlett 1978 Fly gun n04074458). 
Figure 24 : Brevets de pièges captifs
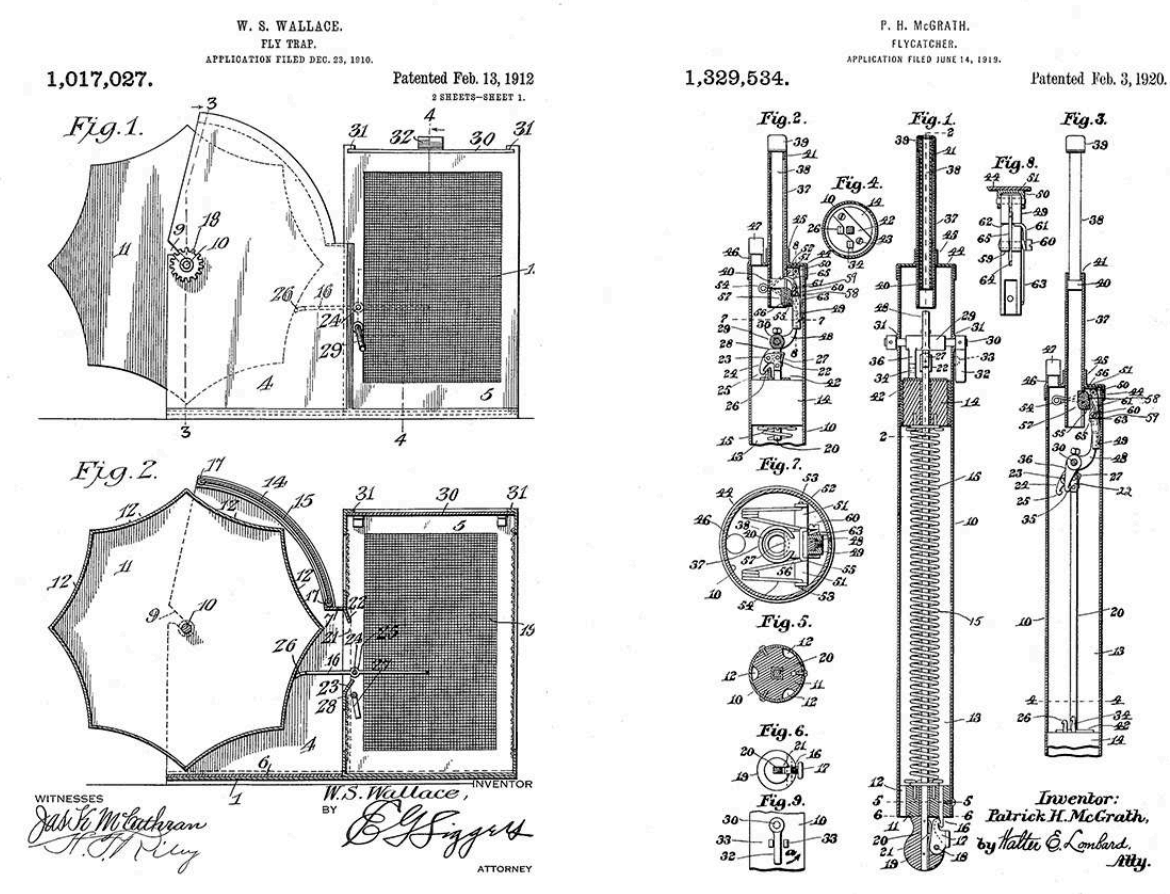

Fly-trap de William S. Wallace (États-Unis, 1912, n¹017027) et Flycatcher de Patrick H. McGrath (États-Unis, 1920, n¹329534)

(c) www.freepatentsonline.com

Des pièges récents, innovants ou inspirés d'autres plus anciens, portent des intentions plus claires. Leurs objectifs - non plus sanitaires mais (bio)éthiques - sont détaillés par leurs concepteurs ou leur notice d'emploi et accompagnent là encore un changement de représentations de l'insecte, comme du vivant plus largement. Ils trouvent leur place « entre le champ des contraintes techniques et celui des possibles symboliques " (Bromberger \& Lenclud 1982:21). L'engin d'Anthony Allen par exemple constitue un piège unique pour capturer les gros insectes, et surtout les araignées (Arachnides), sans les blesser. Par un système de piston des brins sont actionnés pour se saisir de l'animal puis « déplacés dans l'autre sens pour qu'il soit libéré sans mal» (Allen 2003 Insect capturing device $\mathrm{n}^{\circ} \mathrm{U}$ 65574915B1) (Figure 25). De son côté, l'association pour le droit des animaux, Peta, conseille et propose à la vente un piège à insectes et autres petits animaux plus « humain » (c'est-à-dire pour des hommes plus respectueux du vivant), le Humane Bug catcher ${ }^{11}$. Son récipient avec une porte coulissante permet de capturer l'animal et "de le garder en sécurité " avant "de le transporter de la maison à l'extérieur facilement » (Figure 26). 
Figure 25 : Piège « respectueux » et brevet du prototype ETB-UK-NC-2017-01

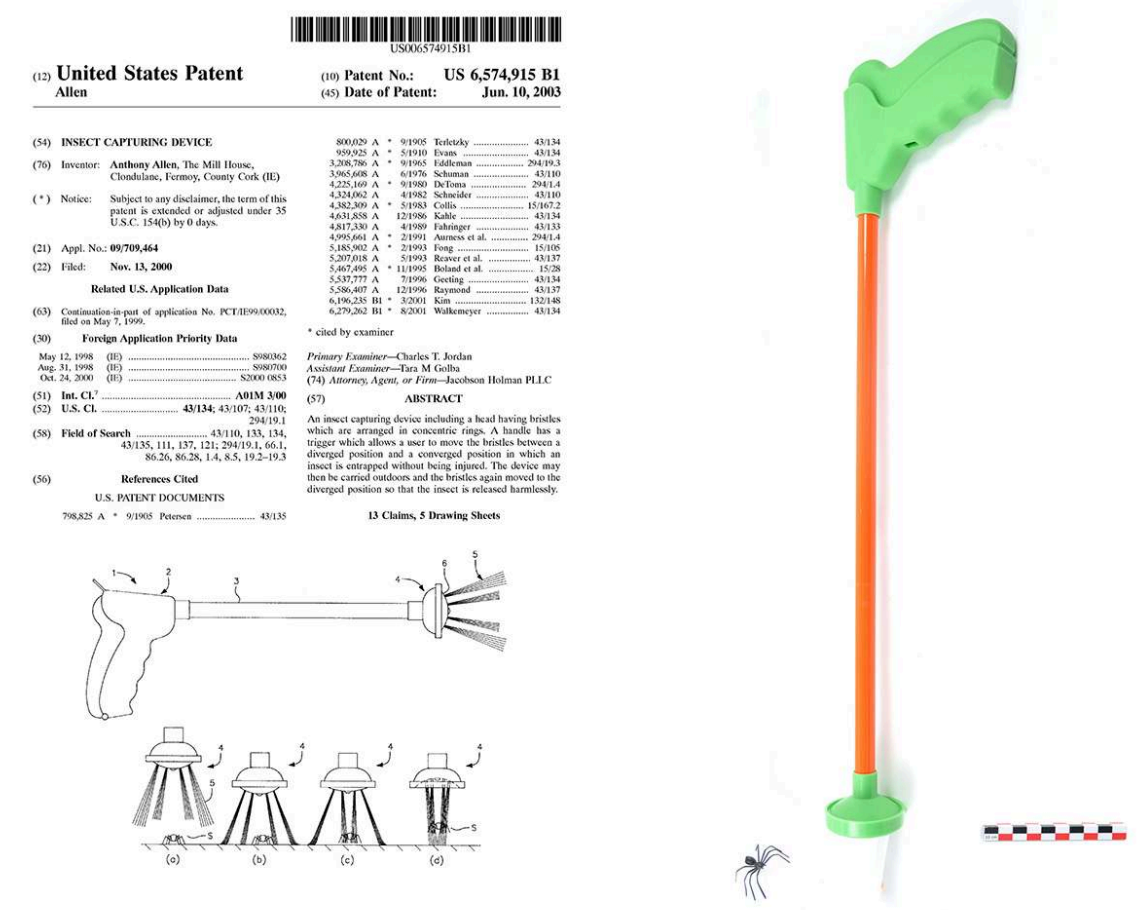

(c) www.freepatentsonline.com (CM. Kourdourli/MNHN

57 - Angleterre

- Insect capturing device de Anthony Allen (États-Unis, 2003, nºS6574915B1) (Source : www.freepatentsonline.com) commercialisé notamment sous le nom de The Buzz Spider Catcher - ETB-UK-NC-2017-01.

- Avril 2017 ; collecteur Nicolas Césard

- Dimensions (cm) 64 x 13

- Nº d'inventaire : ETB-UK-NC-2017-01 
Figure 26 : Attrape arthropodes « respectueux » ETB-DE-NC-2019-01

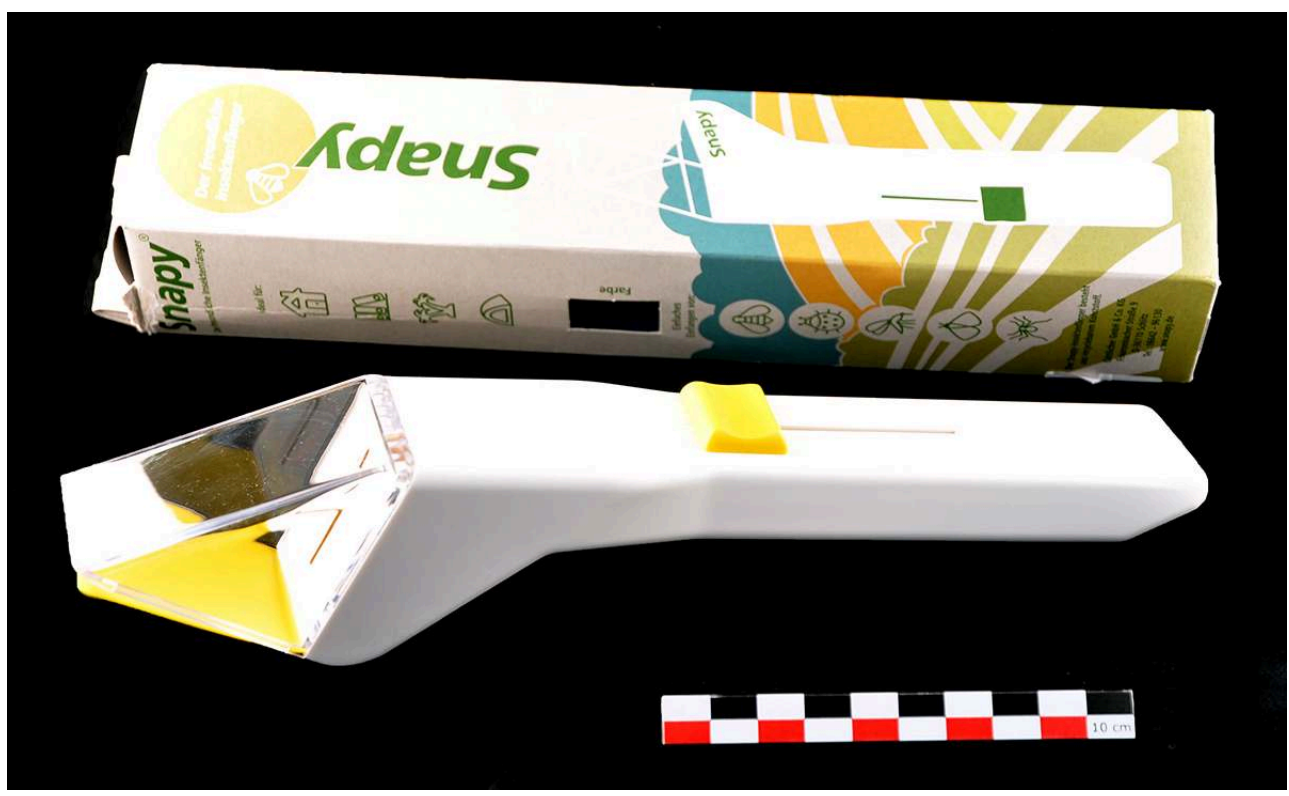

(c) M. Kourdourli/MNHN

- Allemagne

- Snapy. Der Freundliche Insektenfänger. Attrape insectes conseillé par l'association Peta.

- Janvier 2019 ; collecteur Nicolas Césard

Dimensions $(\mathrm{cm}): 26 \times 5$

- $\mathrm{N}^{\circ}$ d'inventaire : ETB-DE-NC-2019-01

Des pièges actuellement disponibles pour tuer ou capturer les mouches, on peut cependant leur préférer une tapette trouée ou sans tamis, un objet détourné capable de satisfaire à la fois la survie de la mouche et l'instinct du chasseur (Figure 27).

Figure 27 : Tapette simple... sans tamis

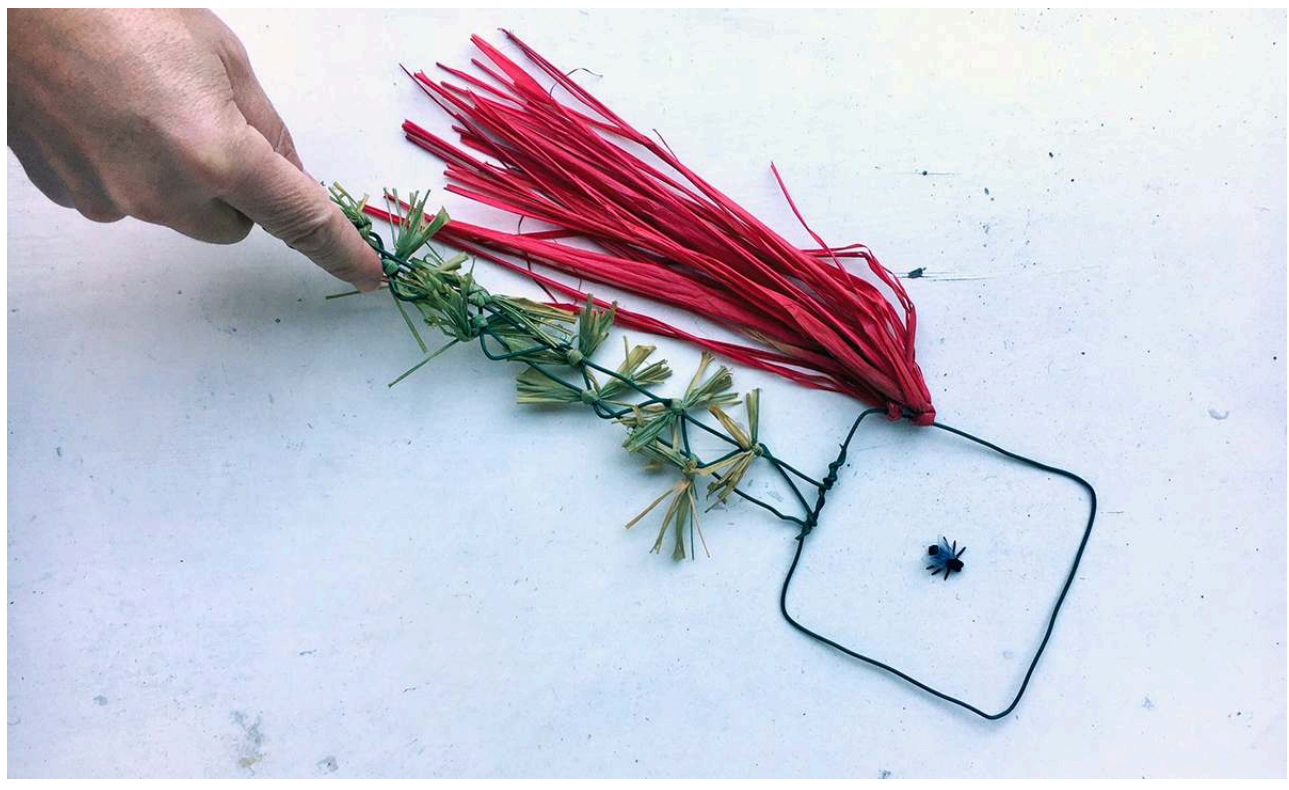

(C) A. Montigny 
- Fabrication Anie Montigny, 1999

- Collection particulière

\section{BIBLIOGRAPHIE}

Bahuchet S. 1971 - Les Gbaya de Centrafrique, étude ethnozoologique. Bulletin de la Société des Amis du Muséum, décembre : 1-4.

Bahuchet S. \& Pujol R. 1975 - Étude ethnozoologique de la chasse et des pièges chez les Isongo de la forêt centrafricaine. In : L'Homme et l'Animal, Premier colloque d'ethnozoologie. Paris, Institut International d'Ethnosciences : 181-192.

Bahuchet S. \& Rameau P. 2016 - Quelques engins de pêche en eau douce d'Afrique centrale. Revue d'ethnoécologie [En ligne], 10, mis en ligne le 31 décembre 2016, consulté le 26 décembre 2019. URL : http://journals.openedition.org/ethnoecologie/2877 ; DOI : 10.4000/ethnoecologie.2877.

Bromberger C. \& Lenclud G. 1982 - La chasse et la cueillette aujourd'hui. Un champ de recherche anthropologique? Études rurales 87 (1) : 7-35.

Card G. \& Dickinson M. 2008 - Visually mediated motor planning in the escape response of drosophila. Current Biology $18: 1300-1307$.

Duperrex M. 2019 - D'une passe à poissons. Milieux et technique en Anthropocène. Techniques \& Culture [En ligne], Varia, mis en ligne le 03 juillet 2019, consulté le 28 octobre 2019. URL : http:// journals.openedition.org/tc/10920

Fraval A. 2008 - La mouche pour cible. Insectes 151 (4) : 35-37.

Haudricourt A-G. \& Bert J-F. 2010 - Des gestes aux techniques: essai sur les techniques dans les sociétés pré-machinistes. Paris, Versailles, Maison des sciences de l'homme, Quae. (Natures sociales).

Mauss M. 1947 - Manuel d'ethnographie. Paris, Payot.

Minnett V. \& Poutanen M.-A. 2007 - Swatting Flies for Health: Children and Tuberculosis in Early Twentieth-Century Montreal. Urban History Review / Revue D'histoire Urbaine 36 (1) : 32-44.

Monestier M. 1999 - Les mouches, le pire ennemi de l'homme. Paris, Le Cherche Midi.

Monod T. 1973 - Contribution à l'établissement d'une classification fonctionnelle des engins de pêche. Bulletin du Muséum national d’histoire naturelle 156 mai-juin. (Écologie générale ; 12).

Rogers N. 1989 - Germs with Legs: Flies, Disease, and the New Public Health. Bulletin of the History of Medicine 63 : 599-604.

Russel E. P. III. 1999 - L.O. Howard promoted war metaphors as a rallying cry for economic entomology. American Entomologist 47 : 74-78.

Simondon G. 1958 - Du mode d'existence des objets techniques. Paris, Aubier.

Uexküll J. von 2010 (1934) - Milieu animal et milieu humain. Paris, Payot. (Rivages). 


\section{NOTES}

1. Les auteurs remercient Nathalie Allain, la famille Benson, Julien Blanc, Myriam Kourdourli, Léo Marquié et Pauline Rameau pour leur aide ou relecture.

2. Nous n'aborderons pas ici ni les insecticides employés à partir des années 1930, ni les produits et les techniques pour éloigner les insectes ou s'en prévenir, qu'ils soient naturels ou nés de la chimie moderne. La collection d'ethnobiologie compte une cinquantaine de tapettes et de pièges à insectes mais également plusieurs pulvérisateurs et souffleurs de différents pays, engins qui consistent à pulvériser le produit directement sur l'insecte ou sur une surface.

3. Article de Rory Galloway, BBC.com, "Why is it so hard to swat a fly ? ", 17/19/2017, http:// bbc.com/news/science-environment-41284065 Accessible en ligne le 15/08/2019

4. Article de Roger Highfield, Telegraph.co/uk, 28/07/2008, "Top five tips for killing flies ». https://www.telegraph.co.uk/news/science/science-news/3350506/Top-five-tips-for-killing-flies.html Accessible en ligne le 15/08/2019.

5. Article de Nakedscientists.com, 10/11/17, « What's the best technique to swat a fly? ». https:// www.thenakedscientists.com/articles/questions/whats-best-technique-swat-fly Accessible en ligne le 15/08/2019.

6. Selon Monestier (1999), dans de nombreuses régions françaises et dans le Poitou en particulier, on se servait pour attirer les mouches de bouquets de fougères fraîches que l'on suspendait au plafond. Les insectes s'y réfugiaient la nuit et tôt le matin. On enserrait alors le tout dans un drap que l'on brûlait.

7. Article de Kevin Dayhoff, 27/08/2017, «In 1914, Westminster fought the Battle of the Flies ». https:// www.carrollcountytimes.com/columnists/features/cc-lt-dayhoff-20170827-story.html Accessible en ligne le $15 / 08 / 2019$.

8. Article de Hope Reeves, nytimes.com, 25/05/2012, «Who Made That Fly Swatter? ». https:// www.nytimes.com/2012/05/27/magazine/who-made-that-fly-swatter.html Accessible en ligne le 15/08/2019.

9. Article de l'Université de Virginie, Historical Collections at The Claude Moore Health Science Library, sans date, "The Invention of the Fly Swatter ». http://exhibits.hsl.virginia.edu/insects/swatter/ Accessible en ligne le 15/08/2019.

10. Article de Kevin Dayhoff, 27/08/2017, "In 1914, Westminster fought the Battle of the Flies ". https://www.carrollcountytimes.com/columnists/features/cc-lt-dayhoff-20170827-story.html Accessible en ligne le 15/08/2019.

11. Voir https://shop.peta.org/humane-bug-catcher.html Accessible en ligne le 15/08/2019.

\section{RÉSUMÉS}

La question est sérieuse et ancienne : comment se débarrasser des mouches ? Plusieurs objets de la collection d'ethnobiologie tentent d'y répondre par différentes méthodes que nous proposons ici de présenter ${ }^{1}$. Considérant les tapettes à mouches comme des pièges, cet inventaire sélectif rend compte du passage dans leur évolution technique et leurs usages d'une approche anthropocentrée à une approche biocentrée.

The question is old and serious: how to get rid of flies? Several objects in the ethnobiology collection attempt to answer it through different methods that we shall hereby present. By 
considering fly swatters as traps, this selective inventory reflects the transition in their technical evolution and uses from an anthropocentric to a biocentric approach.

\section{AUTEURS}

\section{NICOLAS CÉSARD}

Maître de conférences. Laboratoire d'Eco-anthropologie Muséum national d'Histoire naturelle Musée de l'Homme, 17 place du Trocadéro, 75116 Paris ncesard@mnhn.fr

\section{ANIE MONTIGNY}

Maître de conférences. Laboratoire d'Eco-anthropologie Muséum national d'Histoire naturelle Musée de l'Homme, 17 place du Trocadéro, 75116 Paris 